\title{
Glassy systems under time-dependent driving forces: application to slow granular rheology
}

\author{
L. Berthier ${ }^{1}$, L. F. Cugliandolo ${ }^{2}$ and J. L. Iguain ${ }^{3, \star}$ \\ ${ }^{1}$ Laboratoire de Physique, École Normale Supérieure de Lyon, \\ 46 Allée d'Italie, F-69007 Lyon, France and \\ Département de Physique des Matériaux, U. C. B. Lyon 1, \\ 69622 Villeurbanne Cedex, France \\ ${ }^{2}$ Laboratoire de Physique Théorique, École Normale Supérieure, \\ 24 rue Lhomond, F-75231 Paris Cédex 05, France and \\ Laboratoire de Physique Théorique et Hautes Énergies, Jussieu, \\ 4 Place Jussieu, F-75252 Paris Cédex 05, France \\ ${ }^{3}$ Departamento de Física, Universidad Nacional de Mar del Plata, \\ Deán Funes 3350, 7600 Mar del Plata, Argentina
}

July 17, 2021

\begin{abstract}
We study the dynamics of a glassy model with infinite range interactions externally driven by an oscillatory force. We find a well-defined transition in the (Temperature-Amplitude-Frequency) phase diagram between (i) a 'glassy' state characterized by the slow relaxation of one-time quantities, aging in twotime quantities and a modification of the equilibrium fluctuation-dissipation relation; and (ii) a 'liquid' state with a finite relaxation time. In the glassy phase, the degrees of freedom governing the slow relaxation are thermalized to an effective temperature. Using Monte-Carlo simulations, we investigate the effect of trapping regions in phase space on the driven dynamics. We find that it alternates between periods of rapid motion and periods of trapping. These results confirm the strong analogies between the slow granular rheology and the dynamics of glasses. They also provide a theoretical underpinning to earlier attempts to present a thermodynamic description of moderately driven granular materials.
\end{abstract}

${ }^{\star}$ Present address: LPTHE, 4 Place Jussieu, F-75252 Paris Cedex 05, France. LPTENS 00/34, LPTHE 00/36, LPENSL-TH-13/2000. 


\section{Introduction}

In recent years, granular matter has received a growing attention from the physics community [四]. The study of powders is relevant not only because granular materials have many industrial applications but also because it raises many fundamental questions of physical interest.

We call granular matter all many-body systems constituted by grains of macroscopic size. The grains interact via repulsive dissipative forces due to inelastic collisions and static friction. The thermal energy scale is totally negligible with respect to the typical gravitational energy. In the absence of external perturbations, each metastable configuration has an infinite life-time and thermal averaging is meaningless. The static properties of such systems are hence very interesting, the sandpile problem being the paradigm [1].

Powders flow only when energy is supplied externally. This can be done by applying a shear or a vibration. The dynamics of granular matter presents a very rich phenomenology that depends not only on the intensity of the drive, generically called $\Gamma$, but also on the way the granular system is driven [1]. A weak driving force can be provided by applying 'taps' to the systems, as has been done in the pioneering experiments of the Chicago group [2], where the parameter $\Gamma$ is the reduced acceleration of the taps. Recently, Nicolas et al. investigated the dynamics of a powder by sinusoidally shearing it in a weak manner [3]; $\Gamma$ is here the maximal amplitude of the strain. Experiments reveal that the time evolution, in the gently driven situation, is characterized by an extremely slow dynamics [2, 3, (4). In the tapping experiments for instance, the density still evolves after $10^{5}$ taps [2]. When the energy injection is much stronger, the granular matter eventually becomes fluid, it behaves essentially like a dissipative gas and it is described by a hydrodynamic theory that takes into account energy dissipation through the collisions between the grains [1, [5]. In this work we shall focus on the gently driven regime and we shall not address the strongly perturbed situation.

This phenomenology is clearly reminiscent of the behavior of glass forming systems for which the control parameter is the temperature $T$ (or the density $\rho$ ) [6, 0, 8, 9, 10]. At high $T$ (say), the samples are in the liquid or gaseous phases. When $T$ decreases, their dynamics becomes exceedingly slow, and may even appear completely stopped during the experimental time-window. However, at temperatures above but close to the glass transition the relaxation reaches a stationary regime characterized by the decay of all correlations in two steps, the second decay being related to the structural relaxation. At temperatures below the glass transition temperature, the structural relaxation time $t_{r}$ depends on the time spent in the glassy phase (the 'waiting time' $t_{w}$, or 'age') and typically $t_{r} \propto t_{w}$ [11]. A stationary regime cannot be reached experimentally. This is the aging effect which has been observed in a wide spectrum of glassy systems such as plastics [12], spinglasses [13], glycerol [14], dielectric glasses [15], complex fluids [16], phase separating systems [17], etc.

Recently, the similitude in the dynamics of granular matter under vibration and glass forming materials has been rationalized by Liu and Nagel [7]. These authors 
proposed a phase diagram that unifies the physics of glassy systems and granular materials. In its simplest version, the diagram has three axis $(T, \rho, \Gamma)$. The $(T$, $\rho$ ) plane describes the physics of glasses, while the $(\rho, \Gamma)$ plane describes the one of athermal driven systems, like powders or foams. In the low- $T$, high- $\rho$, small- $\Gamma$ region the system is generically jammed or presents glassy features. In this work, we focus on the $(\Gamma, T)$ plane of this phase diagram. The drive axis $\Gamma$ can represent two types of forces: (1) 'shear-like' forces that do not derive from a potential and hence do work on the sample [18]; (2) 'tapping-like' forces that do derive from a potential but do work on the samples when they depend on time. Both modify the dynamic behavior and the goal is to identify how, and to which extent, in a general manner. One possible scenario is that the age of the driven system stabilizes at a power dependent level, typically $t_{r} \propto \Gamma^{-1}$. In the rheological language, this is a shear-thinning behavior. Some examples are given by domain growth under flow [19] or by the non-linear rheology of complex fluids [20], and it has been captured by a number of models [21, 22, 23]. In particular, a (Shear/Temperature) phase diagram for glassy systems has been derived in Ref. [21]. Another scenario, realized in the present paper, is that aging is not stopped, at least in a well-delimitated region of the phase diagram.

The behavior of moderately driven granular matter has received a lot of experimental [2, 3, 目, 24, 25], numerical [9, 26, 27, 28, 29] and theoretical [8, 10, 30, 31, 32, 33, 34, 35, 36, 33, 38] attention. All these studies have demonstrated the glassy nature of granular compaction below a critical amplitude of the drive, $\Gamma^{\star}$. This has been first revealed by the very slow relaxation of the density, but memory experiments [24] and simulations [28, 31] inspired by earlier spin glass studies [11, 13] have also given support to this conclusion.

The relation between granular matter and glassy systems is widely assumed. Indeed, many of the models which have been proposed to study granular compaction are directly adapted from glassy models [8, 26, 227, 28, 29, 36, 37, 38]. Usually, the drive $\Gamma$ in granular matter is related to the temperature $T$ in glasses. However, the assumption that $T=T(\Gamma)$ is a highly non-trivial statement and there is, to our knowledge, no microscopic approach that justifies it. We do not make such an assumption here. In this respect, Mehta et al. [8] have built a phenomenological two-temperature stochastic model based on the observation that the slow granular compaction is basically a two-step process: in this model, a short-time process stands for the fast independent-particle relaxation, while a slow one stands for cooperative rearrangements. The recent experiment of Nicolas et al. [3] clearly proved the existence of these two (uncorrelated) processes. A two-step process, each thermalized with its own temperature is precisely the output of previous studies of the constantly driven dynamics of glassy systems in a thermal bath [18, 21, confirmed by the numerical simulation of a sheared supercooled liquid [39].

In this paper we study the dynamics of a glassy system permanently perturbed by a time-dependent Hamiltonian force [40]. Our aim is to identify which properties correspond to those observed experimentally in granular systems and whether an effective temperature for the slow degrees of freedom is generated in this weakly athermal system. In some sense, our approach is 'orthogonal' to previous ones. 
We do not propose a new model for the slow granular rheology, but rather ask the following simpler question: What is the behavior of a glassy system subjected to periodic driving forces? To answer this question, the $(T, \Gamma, \omega)$ phase diagram is explored. This is done by studying the dynamics of the, by now standard, meanfield glass model, the $p$-spin glass model [11, 43, 44, under a time-dependent driving force. [Albeit its name, that follows from historical reasons, this model represents a structural glass and not a spin glass.] We then discuss how our results have to be interpreted in the context of granular materials.

The paper is organized as follows. In the next Section, we recall the definition and main properties of the $p$-spin glass model. Its behavior under an oscillatory drive, for the choice of parameters $p=2$ and $p \geq 3$, is examined in Sections 3 and 4 , respectively, in two ways: analytical and numerical solutions of the spherical model, and Monte-Carlo simulations of the Ising model. We discuss our results in the context of slow granular rheology in Section 5. Our conclusions close the paper in Section 6 .

\section{Definition and zero-drive behavior of the model}

The spherical $p$-spin glass, when $p=2$, is simply the spherical version of the Sherrington-Kirkpatrick spin glass and it was introduced by Kosterlitz et al. [45] as an exactly solvable model. It is in fact equivalent to the $O(n), n \rightarrow \infty$, model for ferromagnetic domain growth in three dimensions. Its statics [45] and its relaxational dynamics have been extensively studied [46, 47, 48]. The $p \geq 3$ spherical models instead are simplified models for supercooled liquids and glasses, in the sense that they give a theoretical framework to understand both the statics [11, 43, 49] and the non-equilibrium dynamics [11, 50] of glass forming systems.

We study these two cases as generic glassy models, taking advantage of the fact that exact equations for their driven dynamics can be derived.

\section{$2.1 \quad$ Model}

The $p$-spin glass model in its spherical [44 and Ising [43, 51] versions is defined by the Hamiltonian

$$
H_{J}[\mathbf{s}]=\sum_{i_{1}<i_{2}<\cdots<i_{p}} J_{i_{1} i_{2} \ldots i_{p}} s_{i_{1}} s_{i_{2}} \ldots s_{i_{p}},
$$

where the couplings $J_{i_{1} i_{2} \ldots i_{p}}$ are random Gaussian variables with zero mean and variance $\overline{\left(J_{i_{1} i_{2} \ldots i_{p}}\right)^{2}}=p ! /\left(2 N^{p-1}\right)$. The spins $\left\{s_{i}, i=1, \cdots, N\right\}$ may satisfy a global spherical constraint $\sum_{i} s_{i}{ }^{2}(t)=N$, or be Ising variables $s_{i}= \pm 1$. In the spherical version, the driven dynamics is given by the following Langevin equation

$$
\frac{\partial s_{i}(t)}{\partial t}=-\frac{\delta H}{\delta s_{i}(t)}-z(t) s_{i}(t)+f_{i}^{\mathrm{TAPPING}}(t)+\eta_{i}(t)
$$


where the parameter $z(t)$ ensures the spherical constraint and $\eta_{i}(t)$ is the thermal noise, taken from a Gaussian distribution with zero mean and variance $2 k_{\mathrm{B}} T$. [In what follows we use units such that the Boltzmann constant $k_{\mathrm{B}}$ is one.] The thermal bath temperature, $T$, can possibly be zero. In the Ising version one can still write down a continuous Langevin equation by using soft spins and taking the Ising limit at the end of the calculations. The spherical version is simpler to treat analytically while the Ising version is much simpler to deal with using Monte-Carlo simulations, since the spins are bimodal variables.

In order to mimic tapping experiments, a periodic time-dependent Hamiltonian force $f_{i}^{\text {TAPPING }}(t)$ has to be added to the right hand side of the Langevin equation (2). The simplest periodic time-dependence one can think of is a cosine form of period $\tau \equiv 2 \pi / \omega$. Thus, we are lead to add a magnetic field in the Hamiltonian (11),

$$
H_{J}[\mathbf{s}] \rightarrow H_{J}[\mathbf{s}]+\cos (\omega t) \sum_{i=1}^{N} h_{i} s_{i}(t)
$$

In the most realistic numerical experiments, the tapping is modelled by a two-step dilation-relaxation process [9] while in more schematic models, the driving force is not explicitly time-dependent [26, 28, 29, 31]. Two types of spatial dependence of the field will be considered below: constant, $h_{i}=h$, for all sites $i$, and random $\overline{h_{i} h_{j}}=h^{2} \delta_{i j}$. There are however no physical differences between the two situations, since a spatially constant field is as decorrelated from the ground states of the Hamiltonian $H_{J}[\mathbf{s}]$ as a random one.

\subsection{Dynamical equations}

The dynamics of the spherical version of the model is better analyzed in terms of the autocorrelation function $C\left(t, t^{\prime}\right) \equiv \sum_{i} \overline{\left\langle s_{i}(t) s_{i}\left(t^{\prime}\right)\right\rangle} / N$ and the linear response

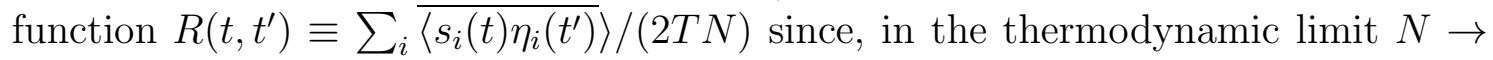
$\infty, C$ and $R$ verify closed Schwinger-Dyson equations which read, for $t>t^{\prime}, 50$

$$
\begin{aligned}
\frac{\partial C\left(t, t^{\prime}\right)}{\partial t}= & -z(t) C\left(t, t^{\prime}\right)+\frac{p(p-1)}{2} \int_{0}^{t} d t^{\prime \prime} C^{p-2}\left(t, t^{\prime \prime}\right) R\left(t, t^{\prime \prime}\right) C\left(t^{\prime \prime}, t^{\prime}\right) \\
& +\frac{p}{2} \int_{0}^{t^{\prime}} d t^{\prime \prime} C^{p-1}\left(t, t^{\prime \prime}\right) R\left(t^{\prime}, t^{\prime \prime}\right)+h^{2} \cos (\omega t) \int_{0}^{t^{\prime}} d t^{\prime \prime} \cos \left(\omega t^{\prime \prime}\right) R\left(t^{\prime}, t^{\prime \prime}\right) \\
\frac{\partial R\left(t, t^{\prime}\right)}{\partial t}= & -z(t) R\left(t, t^{\prime}\right)+\frac{p(p-1)}{2} \int_{t^{\prime}}^{t} d t^{\prime \prime} C^{p-2}\left(t, t^{\prime \prime}\right) R\left(t, t^{\prime \prime}\right) R\left(t^{\prime \prime}, t^{\prime}\right), \\
z(t)= & T+\frac{p^{2}}{2} \int_{0}^{t} d t^{\prime \prime} C^{p-1}\left(t, t^{\prime \prime}\right) R\left(t, t^{\prime \prime}\right)+h^{2} \cos (\omega t) \int_{0}^{t} d t^{\prime \prime} \cos \left(\omega t^{\prime \prime}\right) R\left(t, t^{\prime \prime}\right) .
\end{aligned}
$$

These integro-differential equations are complemented by the equal-times conditions $C(t, t)=1, R\left(t^{+}, t\right)=1$, the symmetry of the correlation, $C\left(t, t^{\prime}\right)=C\left(t^{\prime}, t\right)$, and causality, $R\left(t^{\prime}, t\right)=0$. In deriving these equations, a random initial condition at time $t=0$ has been used, which can be interpreted as an equilibrium configuration 
at infinite temperature. An infinitely fast quench towards the final temperature $T$ is performed at $t=0$ and the evolution continues at subsequent times in isothermal conditions. The energy density $e(t) \equiv N^{-1}\left\langle\overline{H_{J}[\boldsymbol{s}]}\right\rangle$ is related to the constraint $z(t)$ through

$$
e(t)=\frac{1}{p}[T-z(t)]
$$

In Eqs. (đ), the oscillatory field has been chosen to be constant in space.

In the $p=2$ case, the dynamics simplifies considerably since it can be solved directly from the Langevin equation (2). Indeed, this set of $N$ differential equations is diagonalized by using the basis of eigenvectors of the symmetric matrix $J_{i j}$. Denoting $\boldsymbol{\mu}$ the eigenvector associated to the eigenvalue $\mu$, and $s_{\mu} \equiv \boldsymbol{\mu} \cdot \boldsymbol{s}$ and $h_{\mu} \equiv \boldsymbol{\mu} \cdot \boldsymbol{h}$ the projections of the spin and field onto the eigenvectors, one obtains

$$
\frac{\partial s_{\mu}(t)}{\partial t}=(\mu-z(t)) s_{\mu}(t)+h_{\mu} \cos (\omega t)+\eta_{\mu}(t)
$$

Here we have considered, for convenience, a spatially uncorrelated random field. The autocorrelation and response functions become

$$
\begin{aligned}
& C\left(t, t^{\prime}\right)=\int_{-2}^{2} d \mu \rho(\mu)\left\langle s_{\mu}(t) s_{\mu}\left(t^{\prime}\right)\right\rangle, \\
& R\left(t, t^{\prime}\right)=\frac{1}{2 T} \int_{-2}^{2} d \mu \rho(\mu)\left\langle s_{\mu}(t) \eta_{\mu}\left(t^{\prime}\right)\right\rangle,
\end{aligned}
$$

where $\rho(\mu) \equiv \sqrt{4-\mu^{2}} / 2 \pi$ for $\mu \in[-2,2]$, and zero otherwise, is the density of eigenvalues of the random matrix $J_{i j}$. The spherical condition reads $C(t, t)=1$ and after projection, the amplitude of the field $h_{\mu}$ is random with zero mean and variance $\overline{h_{\mu} h_{\mu^{\prime}}}=h^{2} \delta_{\mu \mu^{\prime}}$.

\subsection{Fluctuation-dissipation relation and effective tempera- tures}

We shall be interested in the fluctuation-dissipation theorem (FDT) and its possible modifications. For driven systems, we do not expect this relation to be satisfied. In equilibrium, any correlation function $C\left(t, t^{\prime}\right)=\left\langle O(t) O\left(t^{\prime}\right)\right\rangle$ [we assume, without lose of generality, that $\langle O(t)\rangle=0]$, and its associated linear response $R\left(t, t^{\prime}\right)=$ $\delta\langle O(t)\rangle /\left.\delta f\left(t^{\prime}\right)\right|_{f=0}$, where the perturbation $f$ modifies the Hamiltonian of the system according to $H \rightarrow H-f O$, satisfy the FDT

$$
R\left(t, t^{\prime}\right)=\frac{1}{T} \frac{\partial C\left(t, t^{\prime}\right)}{\partial t^{\prime}} \quad t \geq t^{\prime}
$$

For non-equilibrium systems, a possible extension is [50, 52]

$$
R\left(t, t^{\prime}\right)=\frac{1}{T_{\mathrm{EFF}}\left(t, t^{\prime}\right)} \frac{\partial C\left(t, t^{\prime}\right)}{\partial t^{\prime}} \quad t \geq t^{\prime} .
$$


Naively, this equation is simply a definition of the two-time function $T_{\mathrm{EFF}}\left(t, t^{\prime}\right)$. This extension becomes non-trivial when one realizes that, in the long waiting-time limit of many solvable models, this function only depends on times via the correlation function itself,

$$
T_{\mathrm{EFF}}\left(t, t^{\prime}\right)=\mathcal{T}_{\mathrm{EFF}}\left[C\left(t, t^{\prime}\right)\right]
$$

By extension, it has been proposed that this exact result for solvable, mean-field like models, will also apply to more realistic models with, e.g., finite range interactions. Graphically, a convenient way of checking this ansatz is to represent the integrated response function $\chi\left(t, t^{\prime}\right) \equiv \int_{t^{\prime}}^{t} d t^{\prime \prime} R\left(t, t^{\prime \prime}\right)$ as a function of $C\left(t, t^{\prime}\right)$ at fixed $t^{\prime}$ and parametrized by the time difference $t-t^{\prime}$ [53]. Equation (10) implies that a master curve (i.e. independent of $\left.t^{\prime}\right) \chi(C)$ is asymptotically reached.

In the aging case, three families of models have been found [11: (1) glassy models, for which the $\chi(C)$ curve is a broken straight line with a first piece, from $C=1$ to $C=q_{E A} \equiv \lim _{t \rightarrow \infty} \lim _{t^{\prime} \rightarrow \infty} C\left(t, t^{\prime}\right)$, of slope $-1 / T$ and a second piece, from $C=q_{E A}$ to $C=0$, of slope $-1 / T_{\mathrm{EFF}}, T_{\mathrm{EFF}}$ being finite; (2) 'domain growth models', for which the $\chi(C)$ curve is still a broken straight line with the second piece having $T_{\mathrm{EFF}}=\infty$; (3) spin-glass models, for which the $\chi(C)$ curve has a straight line piece from $C=1$ to $C=q_{E A}$ and a curved piece from $C=q_{E A}$ to $C=0$.

The modification of these plots in a system driven by shear-like forces has been studied at the mean-field level in Refs. [18, 21]. Numerically, the same behavior has been found in a sheared supercooled liquid [39]. Finally, Langer and Liu studied a sheared foam and studied the FDT by comparing the fluctuations of the stress on the boundary and the corresponding compression modulus [54].

At the theoretical level, it was shown in Ref. [52 that the factor $T_{\mathrm{EFF}}$ is indeed a bonafide temperature: it is commonly called 'effective temperature'. A very appealing connection of this factor with the ideas of Edwards [38], in the context of granular matter, and Stillinger-Weber [55] in the context of glass forming liquids are currently being explored [56, 57, 58]. We shall come back to this point below.

Finally we wish to recall a rigorous bound that controls the maximum deviation from FDT that can be observed in an out of equilibrium system with Langevin dynamics [59]. When a time-dependent force of period $\tau$ is applied on the system the bound takes the form

$$
\int_{t_{w}}^{t_{w}+\tau} d s\left(\frac{\partial C(t, s)}{\partial s}-T R(t, s)\right) \leq \sqrt{\left(\mathcal{N} \int_{t_{w}}^{t_{w}+\tau} d s D^{2}(t, s)\right)\left(\frac{W}{N}\right)}
$$

where $W$ is the total work done by the external force $\boldsymbol{h}$ on the system, per period, $W \equiv \int_{t}^{t+\tau} d s\langle\boldsymbol{v}(s) \cdot \boldsymbol{h}(s)\rangle, \mathcal{N}$ is a system dependent numerical factor and $D(t, s)$ is a two-time correlation that in most cases of interest is again bounded by a numerical constant. In particular for the model we treat in this paper, both $\mathcal{N}$ and $D^{2}(t, s)$ equal one. Hence, for this model we have

$$
\int_{t_{w}}^{t_{w}+\tau} d s\left(\frac{\partial C(t, s)}{\partial s}-T R(t, s)\right) \leq \sqrt{\tau\left(\frac{W}{N}\right)} .
$$




\subsection{Zero-drive dynamics: Jammed states and Reynolds di- latancy}

In the absence of a driving force, the model has a dynamical transition at a $(p$ dependent) critical temperature $T_{c}$. For instance, $T_{c}=1$ for $p=2$ and $T_{c} \simeq 0.6123$ for $p=3$. Above $T_{c}$, the equilibration time is finite, and the system reaches equilibrium. In this case, both time-translation invariance (ТTI) and FDT are satisfied and Eqs. (画) reduce to the mode-coupling equation for the so-called $F_{p-1}$ model [11, 43, 60. The relaxation time diverges at $T_{c}$, and below $T_{c}$, the system does not equilibrate with its environment. A quench from the high temperature phase towards the low temperature phase is followed by the aging dynamics described in the introduction: the relaxation time increases with $t_{w}$, which means that TTI is lost. The FDT is modified in the way described in the previous section. Onetime quantities have a slow relaxation - typically power laws and, for instance, the energy density converges to a value $e(t \rightarrow \infty)=e_{t h}(T)$ which is higher than the equilibrium value $e_{e q}(T)$.

From the static point of view [44, 61], $T_{c}$ is the temperature below which the energy landscape becomes fractured into many metastable states. 'Many' means that their number $\mathcal{N}(T, e)$ is such that the thermodynamic limit

$$
\lim _{N \rightarrow \infty} \frac{\ln \mathcal{N}(T, e)}{N}
$$

exists and is finite. The so-called complexity (or 'configurational entropy') $\Sigma(T, e) \equiv$ $\ln \mathcal{N}(T, e)$ is hence extensive in a range $e \in\left[e_{e q}(T), e_{t h}(T)\right]$. The effective temperature $T_{\mathrm{EFF}}$ defined in the previous Section from the FDT violations can be computed directly from the dynamical equations. A remarkable result is that it is also given by 62

$$
\frac{1}{T_{\mathrm{EFF}}}=\left.\frac{\partial \Sigma(T, e)}{\partial e}\right|_{e=e_{t h}}
$$

at $T=0$. [At finite temperature the free energy density replaces the energy density in Eqs. (13) and (14).] This relation is very similar to the thermodynamic definition of temperature and to the definition of Edwards' compactivity in granular systems. We shall come back to these similarities in Section 6 .

The complexity is a useful quantity to understand, for example, the difference between the dynamics of the $p=2$ and $p \geq 3$ models. For $p=2$, one has $e_{e q}=e_{t h}$, and the complexity is irrelevant, $\Sigma=0$. It follows that $T_{\mathrm{EFF}}=\infty$. This case falls in the 'domain growth family' defined in the previous subsection. Instead, if $p \geq 3$, $e_{e q}<e_{t h}, \Sigma>0$ in the region $\left[e_{e q}, e_{t h}\right]$, and $T_{\mathrm{EFF}}$ is finite.

If, instead of a quench from a high temperature at $t=0$, the dynamics starts from one of the metastable states with energy density $e_{e q}<e<e_{t h}$, the system may escape this state, but in a time scale which diverges with $N$. Ergodicity is broken, and the dynamics is blocked. We could call these metastable states the 'jammed states' of the system. (They are known as the TAP states in the spin glass literature 61].) 
When a driving force is applied to the system, the dynamics also depends on the initial state. The driven dynamics following a quench under shear-like forces has been investigated in Refs. [18, 21]. When starting from a low-lying state, and for a small enough driving force, the system remains trapped (jammed). Only by applying a large force will the system escape the deep state and have a dynamics like the one following a quench [18]. Hence, the system becomes able to move only by raising its energy density: this is, in the glassy context, the Reynolds dilatancy effect [1, 10].

The effect of these trapping states on the dynamics following a quench are beyond the mean-field approximation, Eqs. (14). We propose to investigate this interesting aspect by simulating in Section 4.3 the Ising version of the model keeping finite the number $N$ of interacting spins, so that jammed states will be dynamically accessible by the system.

\section{Driven spherical Sherrington-Kirkpatrick model}

In this Section we study analytically the driven dynamics of the $p=2$ case.

\subsection{Existence of a dynamic transition}

Let us first study the $p=2$ case at $T=0$. The Langevin equation (6) can be integrated out; this yields

$$
s_{\mu}(t)=\frac{e^{\mu t}}{\Omega(t)}\left[1+h_{\mu} \int_{0}^{t} d t^{\prime} \cos \left(\omega t^{\prime}\right) e^{-\mu t^{\prime}} \Omega\left(t^{\prime}\right)\right]
$$

where the function

$$
\Omega(t) \equiv \exp \left[\int_{0}^{t} d t^{\prime} z\left(t^{\prime}\right)\right]
$$

has been defined. The spherical constraint determines $\Omega(t)$ through the equation

$$
\Omega(t)^{2}=f(t)+h^{2} \int_{0}^{t} d t^{\prime} \int_{0}^{t} d t^{\prime \prime} f\left(t-\frac{t^{\prime}+t^{\prime \prime}}{2}\right) \Omega\left(t^{\prime}\right) \Omega\left(t^{\prime \prime}\right) \cos \left(\omega t^{\prime}\right) \cos \left(\omega t^{\prime \prime}\right),
$$

with $f(t)$ given by

$$
f(t) \equiv \int_{-2}^{2} d \mu \rho(\mu) e^{2 \mu t} .
$$

The autocorrelation and response functions, together with the energy density, read

$$
\begin{aligned}
C\left(t, t_{w}\right)= & \frac{1}{\Omega(t) \Omega\left(t_{w}\right)}\left[f\left(\frac{t+t_{w}}{2}\right)\right. \\
& \left.+h^{2} \int_{0}^{t} d t^{\prime} \int_{0}^{t_{w}} d t^{\prime \prime} \cos \left(\omega t^{\prime}\right) \cos \left(\omega t^{\prime \prime}\right) \Omega\left(t^{\prime}\right) \Omega\left(t^{\prime \prime}\right) f\left(\frac{t+t_{w}-t^{\prime}-t^{\prime \prime}}{2}\right)\right], \\
R\left(t, t_{w}\right)= & \frac{\Omega\left(t_{w}\right)}{\Omega(t)} f\left(\frac{t-t_{w}}{2}\right), \quad e(t)=-\frac{z(t)}{2}=-\frac{1}{2} \frac{d \ln [\Omega(t)]}{d t} .
\end{aligned}
$$




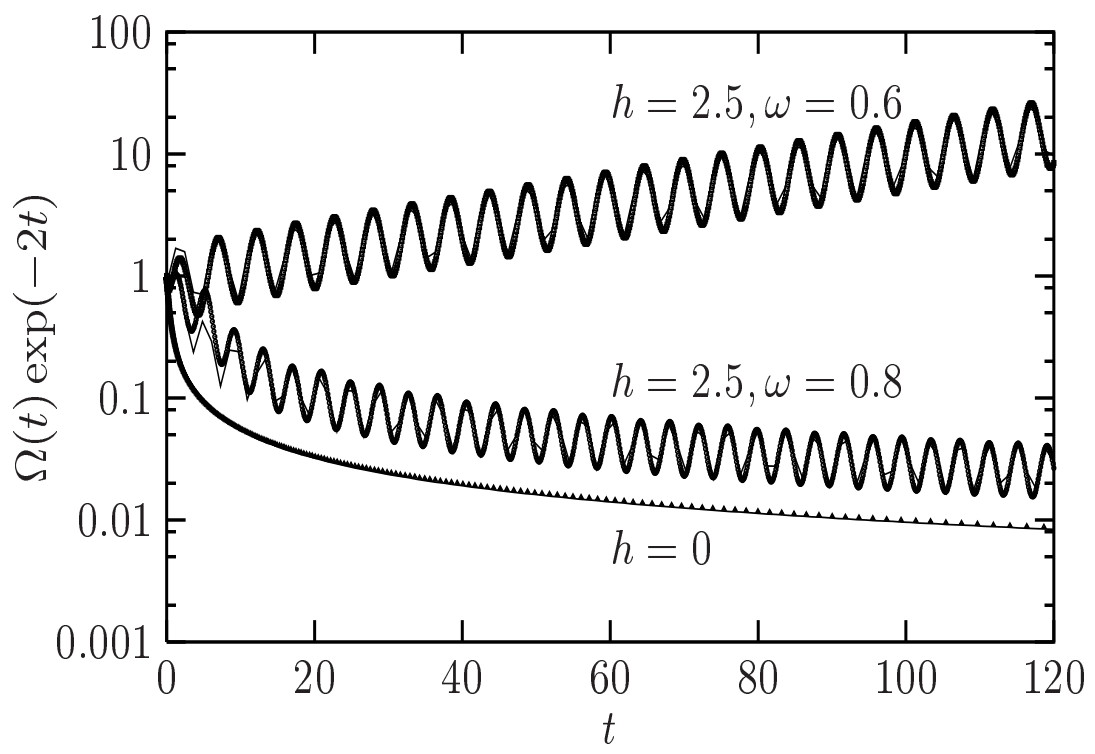

Figure 1: The function $\Omega(t) \exp (-2 t)$ in the three cases $h=0$ and $h=2.5$ with $\omega=0.8$ (glassy phase) and 0.6 (liquid phase), from bottom to top. The points are the direct integration of Eq. (17), while the full lines are fits to Eqs. (20). Except at very short times, the approximate forms in Eqs. (20) yield the correct behavior.

Equation (17) determines $\Omega(t)$ and, consequently, all dynamic quantities. Unfortunately, it cannot be completely solved analytically except in some asymptotic limits.

Two special cases have been studied previously, namely the zero field behavior of the model, and the case of a dc field. In the former, as discussed in Section 2 , there is no finite equilibration time below $T_{c}=1$ and the system ages forever 46 , 477. In the presence of a dc magnetic field of amplitude $h$ [48], Eq. (17) yields $\Omega(t) \sim \exp \left(\lambda^{0}(h) t\right)$, where $\lambda^{0}(h) \equiv\left(2+h^{2}\right) / \sqrt{1+h^{2}}$. The main effect of the field is to introduce a new time scale $t_{r}{ }^{0}(h) \equiv\left[\lambda^{0}(h)-2\right]^{-1}$ in the problem. For times $t_{w} \ll t_{r}{ }^{0}(h)$ after the quench, aging is observed as in the zero-field case, whereas at later times $t_{w} \gg t_{r}^{0}(h)$, the system has reached its equilibrium state in a field: aging is interrupted. The energy density $e(t)$ converges to $e_{\infty}(\omega=0, h)=$ $-\left(2+h^{2}\right) /\left(2 \sqrt{1+h^{2}}\right)$.

At finite $\omega$, the numerical solution of Eq. (17) displayed in Fig. 11 suggests the following asymptotic behavior:

$$
\begin{aligned}
h<h^{\star}(\omega) & \Omega(t) \sim c \frac{e^{2 t}}{t^{3 / 4}}(b \cos (2 \omega t+\phi)+1), \\
h>h^{\star}(\omega) & \Omega(t) \sim c^{\prime} \exp [\lambda(\omega, h) t+a \cos (2 \omega t+\phi)],
\end{aligned}
$$

where $c, c^{\prime}, a, b<1$ and $\phi$ are numerical constants, and with $\lambda(\omega, h)>2$. This means that if the amplitude of the driving force is sufficiently small, the ac field does not introduce a new time scale and a full aging behavior is observed. For stronger 
amplitudes of the field, a time scale defined by

$$
t_{r}(\omega, h) \equiv[\lambda(\omega, h)-2]^{-1}
$$

is generated and the relaxation time becomes finite: aging is stopped by the driving force. There is hence a critical field $h^{\star}$ separating these two different regimes. It is clear from Fig. 11 that the agreement between Eqs. (20) and the numerical solution is very good, after a short transient.

In the following we characterize more precisely these two phases as well as the transition between them.

\subsection{The transition line in the plane $(\omega, h)$}

We have seen that, at fixed pulsation $\omega$, there exists a well-defined transition line where the relaxation time in an ac field diverges, allowing to distinguish between a 'glassy' and a 'liquid' state. We anticipate the discussion of the last section to emphasize that this transition is a non-equilibrium phase transition which is hence of a different nature that the transition taking place at $T_{c}$ in the absence of the driving force.

The transition line $h^{\star}(\omega)$ may be understood and estimated from a simple physical argument. At $\omega=0$, the dc field introduces a finite relaxation time $t_{r}{ }^{0}(h)$. The most naive requirement for the system to keep a finite relaxation time in an ac field of period $\tau$ is given by $t_{r}{ }^{0}(h) \lesssim \tau$. The transition line is then estimated by the relation

$$
t_{r}^{0}\left(h^{\star}\right) \simeq \tau \quad \Leftrightarrow \quad \omega \propto\left[\frac{2+h^{\star 2}}{\sqrt{1+h^{\star 2}}}-2\right] .
$$

A more refined computation can also be performed. Using the fact that, when $h>h^{\star}, \Omega(t)$ is given asymptotically by Eq. (20) with $\lambda>2$ allows us to neglect the term $f(t)$ in the right hand side of Eq. (17); thus

$$
\Omega(t)^{2} \simeq h^{2} \int_{-2}^{2} d \mu \rho(\mu) e^{2 \mu t}\left[\int_{0}^{t} d t^{\prime} \cos \left(\omega t^{\prime}\right) \Omega\left(t^{\prime}\right) e^{-\mu t^{\prime}}\right]^{2}
$$

Inserting the form $\Omega(t)=\exp (\lambda t+B(t))$, and formally integrating by parts yields

$$
e^{B(t)}=h^{2} \int_{-2}^{2} d \mu \rho(\mu)\left[\Re\left(e^{i \omega t} \sum_{k=0}^{\infty} \frac{(-1)^{k}}{(\lambda(\omega, h)-\mu+i \omega)^{k+1}} \cdot \frac{d^{k} e^{B(t)}}{d t^{k}}\right)\right]^{2} .
$$

Since the function $B(t)$ is periodic with angular velocity $2 \omega$, this corresponds to a development in powers of $\omega$. In the small frequency limit, the equation can be closed by keeping only the leading terms of the development, and this yields a relation between $(\lambda, \omega, h)$ :

$$
\frac{\pi}{h^{2}}=\int_{-1}^{1} d x \frac{\sqrt{1-x^{2}}}{(\lambda-2 x)^{2}+\omega^{2}}
$$




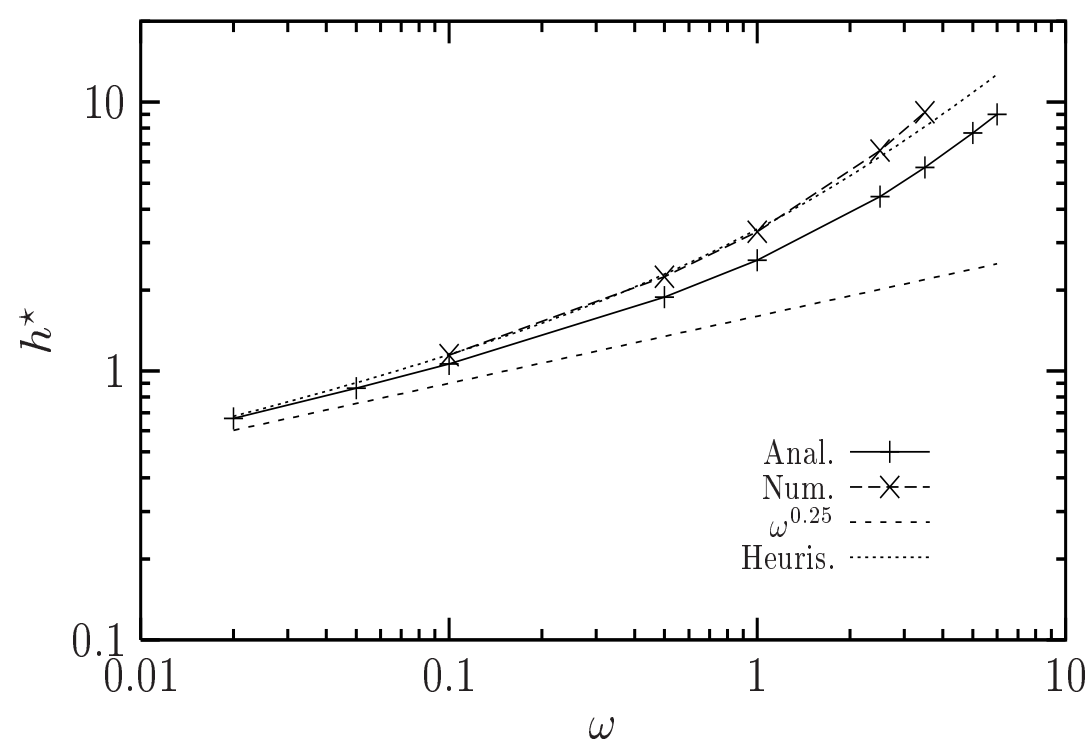

Figure 2: The transition line $h^{\star}(\omega)$, estimated by analytical, numerical and heuristic tools, together with the small frequency behavior $h^{\star} \propto \omega^{0.25}$.

from which we estimate the transition line where the time scale $t_{r}(\omega, h)$ given in Eq. 211) diverges. This is equivalent to the condition $\lambda\left(\omega, h^{\star}\right)=2$. It is easily shown that in the small frequency limit, the scaling $t_{r}{ }^{0}\left(h^{\star}\right) \simeq \tau$ is recovered, in accordance with our heuristic argument, Eq. (22). In Fig. 2, the analytic estimate for the transition line $h^{\star}(\omega)$ is compared with its direct numerical estimation, and with our heuristic argument. Also plotted is the small frequency behavior of the critical field, $h^{\star} \propto \omega^{0.25}$.

Moreover, Equation (25) implies

$$
\lim _{\omega \rightarrow 0} \lambda(\omega, h)=\lambda^{0}\left(\frac{h}{\sqrt{2}}\right),
$$

which means that, in the limit $\omega \rightarrow 0$, the field acts as a constant field with an amplitude given by its root mean square value, which is physically reasonable. Importantly enough, this means that the effect of a dc field with $\omega$ strictly zero and the limit of an ac field with vanishing frequency are different. In the case $p=2$ this feature has no effect in the value of the transition field $h^{\star}$ since it vanishes in both cases. However, when $p \geq 3$ we shall find a non trivial consequence of this result (see Section 田).

\subsection{Below the transition $h<h^{\star}(\omega)$ : aging in an ac field.}

Below the transition, Eq. (20) shows that the energy density slowly converges towards its asymptotic value $e_{\infty}\left(\omega, h<h^{\star}\right)=-1$ as a power law $e(t)-e_{\infty} \sim t^{-1}$ with a superimposed oscillation at a frequency $\omega / \pi$. This asymptotic value is independent of $h$. A slow (here a power law) convergence of one time quantities is typical 


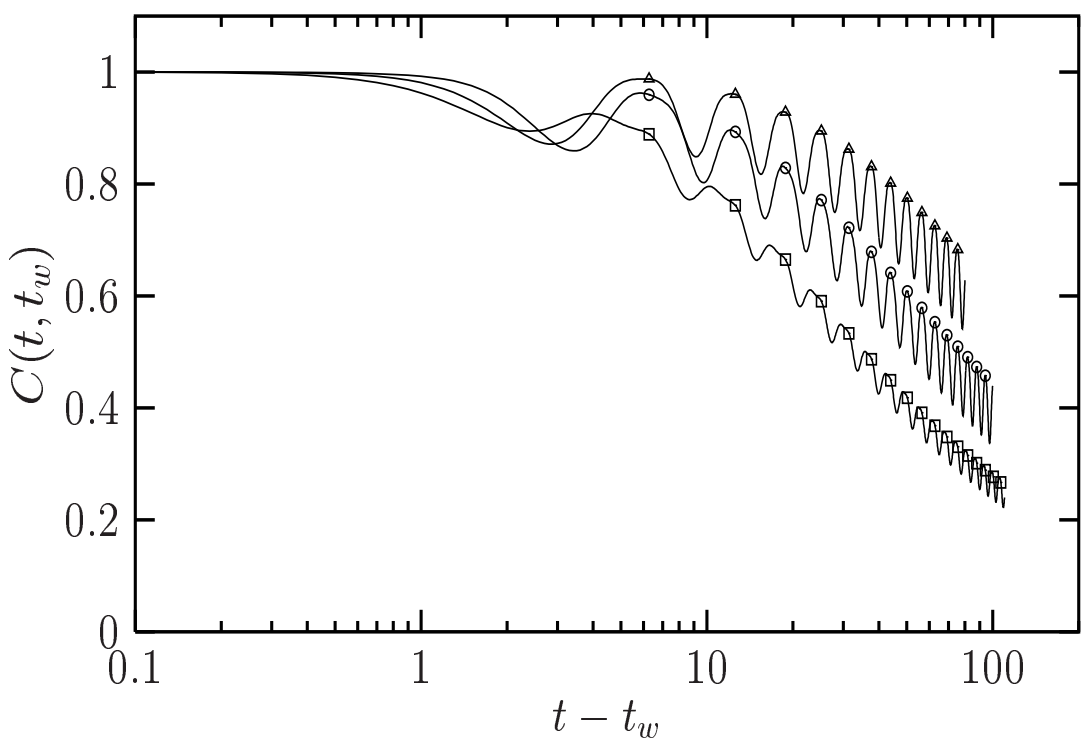

Figure 3: Correlation function of the $p=2$ model at $T=0$ for $h=0.5, \omega=1$ and waiting times $t_{w}=5,10,20$, from bottom to top. The points fall on the discrete times $t=n \tau$, with $n$ an integer.

of aging systems [11]. The angular velocity $2 \omega$ is expected since Eq. (6i) remains unchanged by the transformation $t \rightarrow t+\pi / \omega ; s_{\mu} \rightarrow-s_{\mu} ; z \rightarrow z$. The constraint $z(t)$, and the energy density, are then $\pi / \omega$-periodic.

That TTI is also lost is demonstrated by looking at two-time quantities, typically correlation functions. The behavior of $C\left(t, t_{w}\right)$ is represented in Fig. 3. This figure also shows the interesting feature that the dynamics can be decomposed into two well-separated time scales. At short time separation $t-t_{w}$, the time-scale for the approach to the plateau at $C=q_{\mathrm{EA}}$ (note that $q_{\mathrm{EA}}=1$ at $T=0$ ) does not depend on $t_{w}$, whereas the decay from the plateau towards zero arises in a second time scale that clearly depends on $t_{w}$. During the waiting-time dependent decay the curves have oscillations. Quantitatively, the behavior of the correlation function is entirely dominated at long times by the term $C\left(t, t_{w}\right) \sim f\left(\frac{t+t_{w}}{2}\right) /\left(\Omega(t) \Omega\left(t_{w}\right)\right)$. This implies that, apart from the oscillation, the correlation scales as $t / t_{w}$ for $t \gg t_{w}$; we have numerically checked this point.

In tapping experiments, data are obtained for times of the form $t=n \tau$, where $n$ is an integer. We have represented these data by points in Fig. 3, and the experimental measurements would be very similar to the usual aging case.

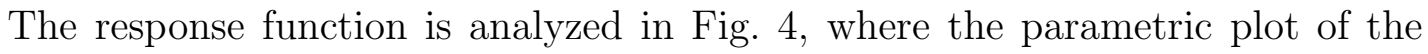
integrated response against the correlation is built. As expected, there are strong violations of the FDT. These violations are however similar to those encountered in the relaxational case, in the sense that there are two distinct behaviors, depending on the time scale considered. For a time separation $t-t_{w} \ll t_{w}$, FDT is not satisfied, and no effective temperature can be defined. For the second time scale $t \gtrsim t_{w}$ and 


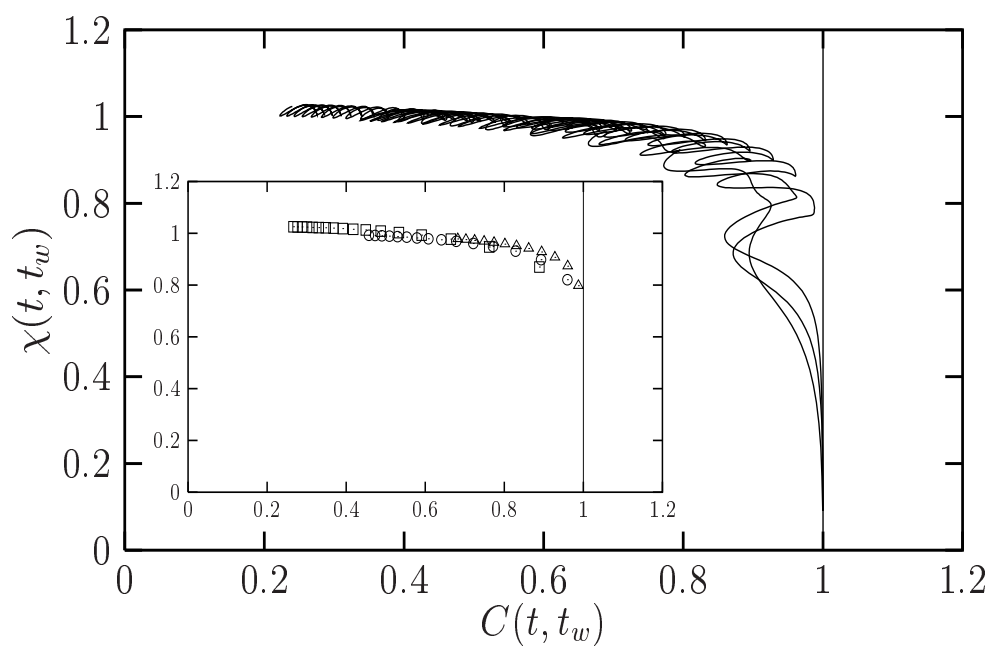

Figure 4: Parametric plot of the integrated response-correlation of the $p=2$ model at $T=0$ for $h=0.5, \omega=1$ and waiting times $t_{w}=5,10$ and 20. For the FDT to be satisfied, the points have to lie on the vertical line. The inset represents the same data, but for times $t=n \tau$; the symbols are the same as in Fig. 3 .

longer, on the contrary, all points fall nicely on a straight line, with a superimposed oscillation. The bound (12) shows however that the deviation from FDT can only be significant at large times, when both $\partial C\left(t, t_{w}\right) / \partial t_{w}$ and $R\left(t, t_{w}\right)$, integrated over a period, are small. This is confirmed by Fig. 4 .

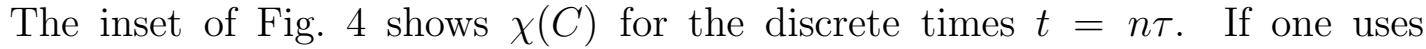
this stroboscopic measurement, it remains clear that FDT modifications are well accounted for by the ansatz (10). Analytically, neglecting as above the second term in the correlation function, the effective temperature defined through Eq. (9) scales as $T_{\mathrm{EFF}} \sim t_{w}^{1 / 2} \rightarrow \infty$. An infinite effective temperature is also present in the relaxational dynamics of this model, and is typical of domain growth models.

With these simple considerations we have argued that the effect of a small ac field does not change the aging behavior of this model at $T=0$. This is to be confronted to the effect of non-Hamiltonian perturbations that change the aging scaling, as seen for instance in the correlation function that becomes $C\left(t, t_{w}\right) \sim f\left(\exp \left(\sqrt{t_{w}}-\right.\right.$ $\sqrt{t})$ ) [18. At non-zero temperature, shear-like forces introduce a finite relaxation time after which the dynamics is stationary. We have not solved analytically the $p=2$ model at finite temperature in an ac field, but the numerical solution of the full equations suggests that there is a finite region of the phase diagram $(T, h, \omega)$ in which aging effects survive. This explicitly shows that the effect of shear and tapping are rather different. 


\subsection{Above the transition: driven steady state.}

Above the transition, the situation is simpler. After a time scale $t_{r}(\omega, h)=[\lambda(\omega, h)-$ $2]^{-1}$ the slow dynamics is lost. The relation (25) implicitly determines $\lambda$, which is an increasing function of the amplitude $h$ of the driving force. In the stationary state, the energy density oscillates with an angular velocity $2 \omega$ around its asymptotic value $e_{\infty}\left(\omega, h>h^{\star}\right)=-\lambda(\omega, h) / 2$. The behavior of the correlation is dominated by the second term in Eq. (19)). This shows that it decays towards zero in a time scale of order $t_{r}(\omega, h)$ : there is no more aging.

\section{Behavior for $p \geq 3$}

Going beyond the solution of the unperturbed case [50] to solve the set of coupled integro-differential equations (4) analytically is a very hard task. In order to illustrate the main properties of the solution, we solved Eqs. (四) numerically, by constructing the two-time solution step by step in time. We first demonstrate that also in the case $p \geq 3$, there exists a transition line below which glassy properties of the undriven model persist, and study then the FDT-violations in both phases.

\subsection{Evidences for a dynamical transition}

Let us recall first the effect of a dc magnetic field of amplitude $h$ on the system [63]. In contrast to the spherical Sherrington-Kirkpatrick, the spin glass phase may exist in a dc magnetic field. There are both a dynamic and a static transition between the spin glass and the paramagnetic phases. At $T=0.2$, the dynamic transition takes place at $h^{\star}(\omega=0) \simeq 1.1$ [63].

As in the case $p=2$, we begin our study by focusing on the behavior of the energy density $e(t)$. It is displayed in Fig. 5 at temperature $T=0.6$ and angular velocity $\omega=1$, for different field amplitudes. The long time behavior of $e(t)$ indicates that the transition occurs for a field $0.6<h^{\star}<1.0$ at this frequency. The asymptotic value of the energy density slightly increases below the transition, and reaches a much larger value, when $h>h^{\star}$.

In the following we concentrate on the temperature $T=0.2 \simeq 0.32 T_{c}$, i.e. well below the zero-field transition. We focus first on the dependence of the two-time autocorrelation functions on the amplitude of the applied field. In Fig 6-a, the field is $h=0$, and we observe the usual aging [50]. It is then clear that aging is still present if the field is not too strong, $h=0.1$ and $h=1$ in Figs. 6-b and c, whereas at a stronger field, $h=2$ in Fig. 6-d, the correlation very rapidly tends to zero, with a superimposed oscillation. These observations reinforce the evidence in favour of a dynamic transition which, at $T=0.2$, occurs at a field strength $1<h^{\star}<2$, for $\omega=1$.

We turn now to the question of identifying the critical line in the $\omega$ direction. Fig. (7) shows the auto correlation function at fixed amplitude of the applied field and several values of the angular velocity. In Fig. (7-a, the frequency is zero, and aging is observed with a two-step decay of the correlation. Note that the second decay 


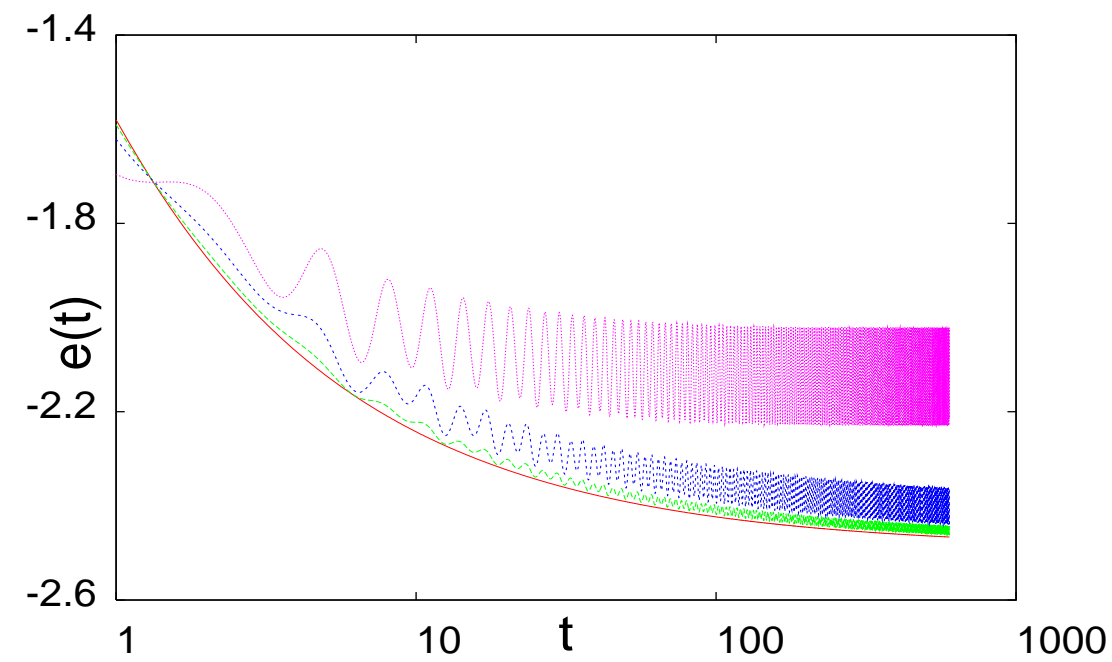

Figure 5: Time dependence of the energy density under an ac field with amplitude $h=0,0.3,0.6,1$ (from bottom to top) and angular velocity $\omega=1$. The curve for $h=1$ approaches rapidly an asymptote, whereas $e(t)$ still evolves at large times for the other cases.

is towards a value $q_{0}>0$, as opposed to the zero-field case 63. The frequency in increased in Figs. 가, c,d. At intermediate frequency, Fig. 가, aging is suppressed. When $\omega$ is further increased, Figs. 7-c,d, it is clear that the glassy phase is reentrant.

It is of course very difficult to determine the transition line $h^{\star}(\omega)$ with accuracy, by the numerical solution of the dynamical equations. We are hence not able to draw a figure similar to Fig. 2 for $p \geq 3$. We show in Fig. 8 a schematic representation of this critical line with the feature, already encountered for $p=2$, that the limit $\omega \rightarrow 0$ is peculiar. From the numerical solution at $\omega>0$ it seems that

$$
\lim _{\omega \rightarrow 0} h^{\star}(T, \omega)<h^{\star}(T, \omega=0) .
$$

This is represented in Fig. 8 and explains the reentrance of the glassy phase described in Fig. 7, when the frequency increases. Note however that we cannot numerically discard the possibility of a non-monotonous, but smooth, behavior of the critical line at $\omega<0.1$.

\subsection{Fluctuation-dissipation theorem}

We turn now to the study of the FDT. In Fig. 9 we show the evolution of the $\chi$ vs $C$ plots with $t_{w}$. In Fig. 9-a, the system is not in the glassy phase. Strong violations of the FDT are observed, but no effective temperature can be defined. The system is athermal.

In Fig. 9-b, on the contrary, the system is in its glassy phase. One recovers then a $\chi(C)$ curve that is very similar to the one in zero field. The first part is almost a straight line with slope $-1 / T$ while the second decay follows a temperature $-1 / T_{\mathrm{EFF}}$. 

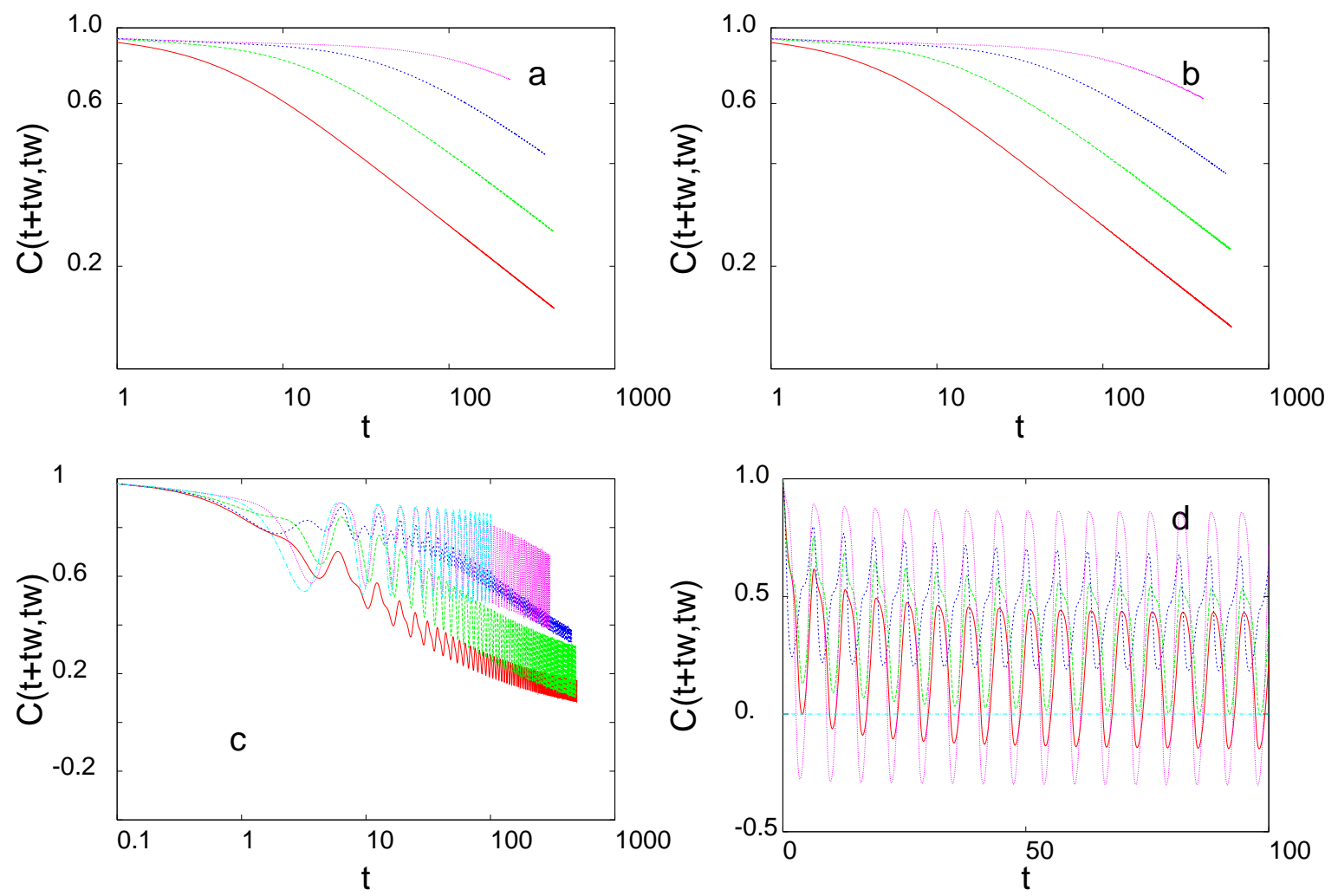

Figure 6: Each panel shows the auto-correlation $C\left(t+t_{w}, t_{w}\right)$ as a function of $t$, for four values of the waiting-time $t_{w}=3,12,49,198$ at $T=0.2$. The angular velocity of the applied field is $\omega=1$. In panels a to c the scale is logarithmic, the amplitudes of the applied fields are $h=0,0.1,1$ and the system is in its glassy phase. In panel $\mathrm{d}$ the scale is linear, $h=2$ and the slow dynamics is suppressed.

As mentioned in Section 3, the fact that for small time scales, the FDT is nearly satisfied results directly from the bound (12).

The non-trivial outcome of this study is the fact that a well-defined $T_{\mathrm{EFF}}$, which exists in the non-driven system, may still be defined below the dynamical transition, in presence of the drive. A stroboscopic construction, as the one in Section 3.3, will yield a perfect straight line that defines $T_{\mathrm{EFF}}$ unambiguously.

The actual value of the effective temperature may depend on various parameters. We have searched for dependences on the frequency and amplitude of the ac field. Within the range of parameters we could numerically explore, we have not observed large dependences. Figure 10 display the $\chi$ vs $C$ curves for two choices of field amplitudes, and for two choices of angular velocity at fixed field amplitude: the dependence is indeed weak.

\subsection{Beyond mean-field: Effect of trapping states}

In this Section we present results from a numerical simulation of the Ising version of the $p$-spin model in an ac field, in the particular case $p=3$. We focus on 

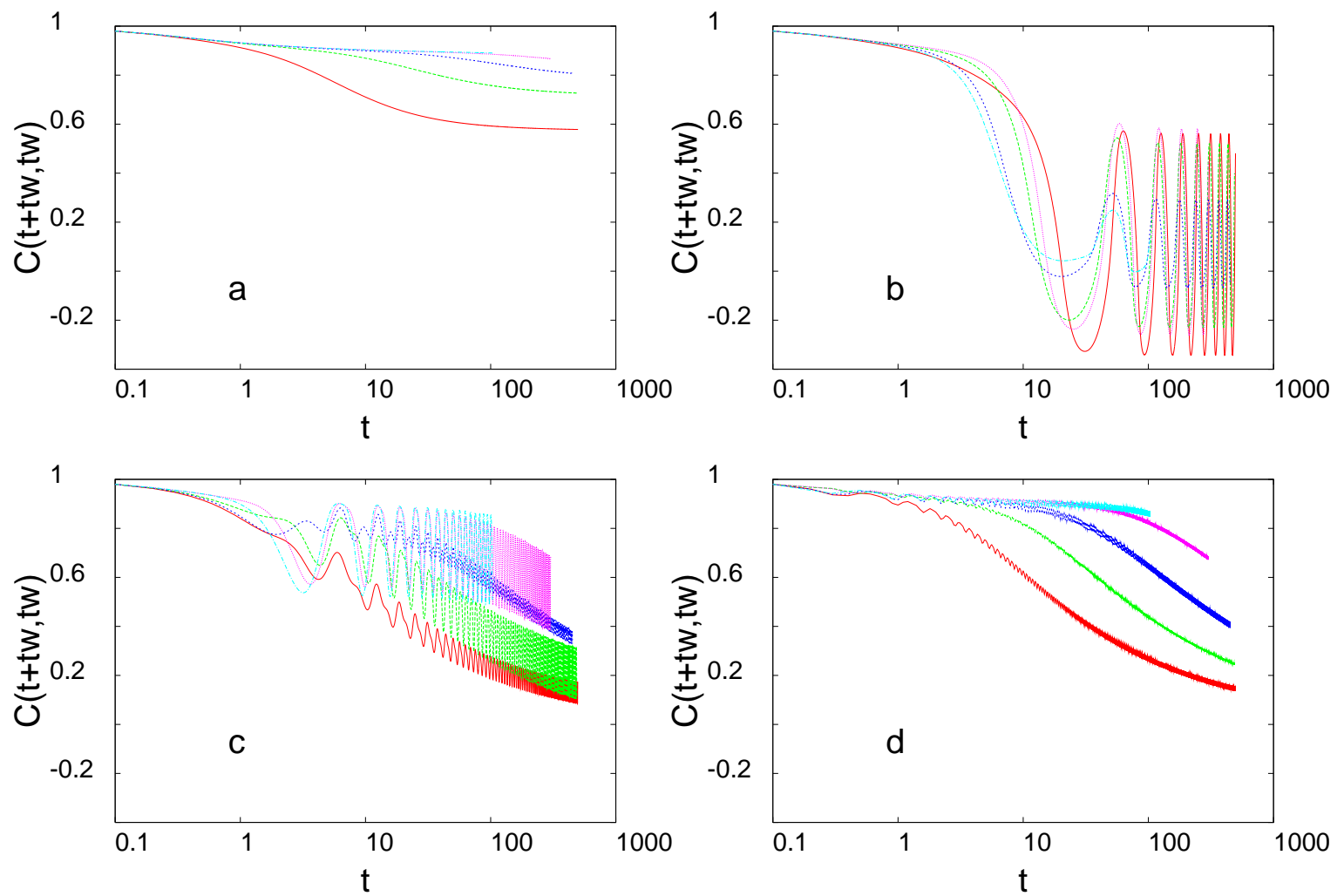

Figure 7: Each panel shows the auto-correlation $C\left(t+t_{w}, t_{w}\right)$ as a function of $t$, for four values of the waiting-time $t_{w}=3,12,49,198$ at $T=0.2$. The strength of the applied field is $h=1$ and the frequencies are $\omega=0,0.1,1,10$ from panel a to panel d. The transition in an ac-field of amplitude $h=1$, from the liquid to the glassy phase, occurs at a frequency $0.1<\omega_{c}<1$.

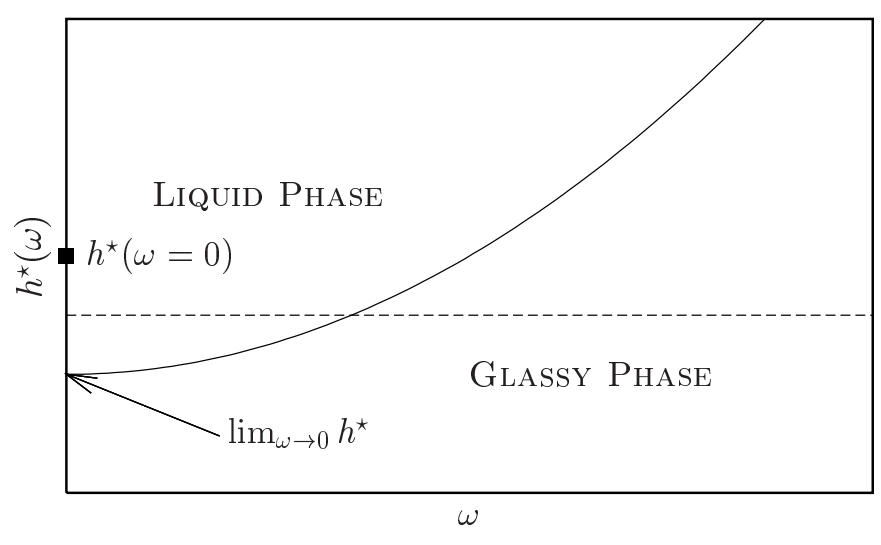

Figure 8: The full line is a schematic representation of the transition line $h^{\star}(\omega)$ for $p \geq 3$. The angular velocity in the four panels in Fig. 7 move from left to right along the horizontal dashed line (see the text for more details). 

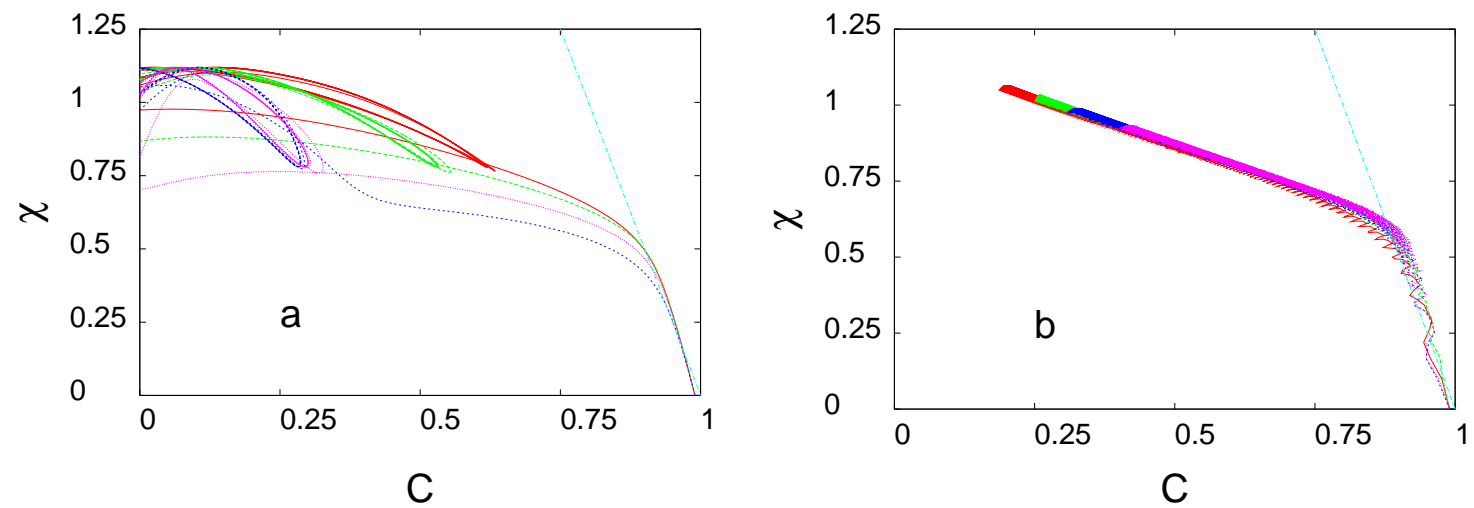

Figure 9: The $\chi(C)$ curves at $T=0.2$ with an applied ac-field of strength $h=1$ and frequencies $\omega=0.1$ (liquid phase) and $\omega=10$ (glassy phase), for different waiting times $t_{w}=6,12,24,49$.
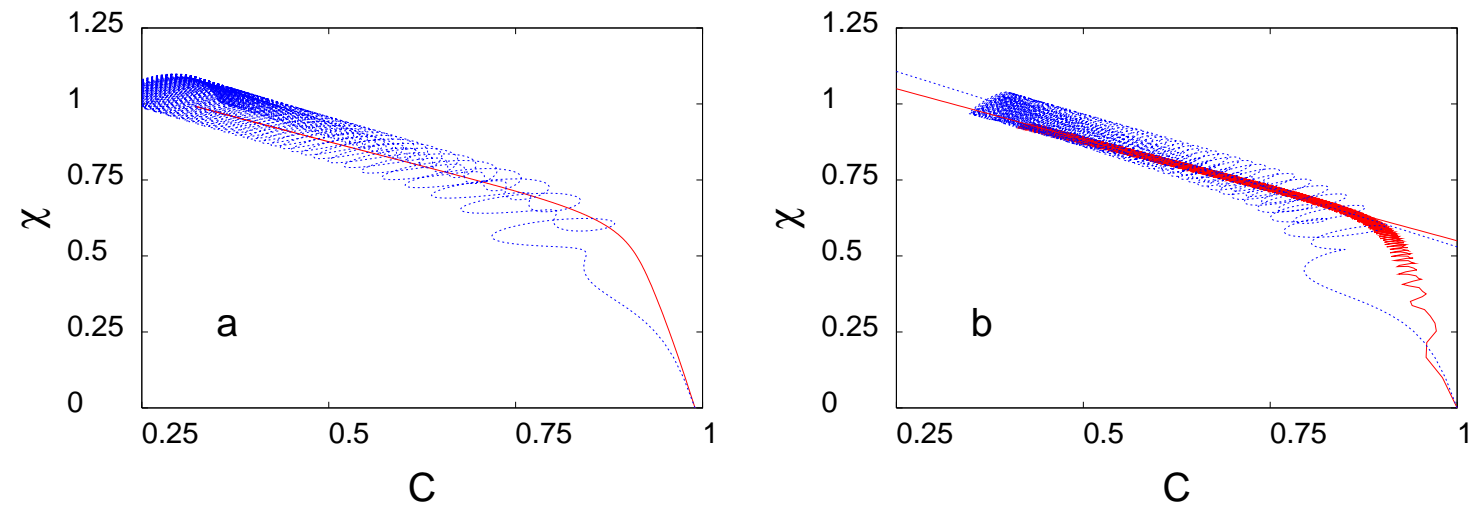

Figure 10: The $\chi(C)$ curves at $T=0.2$. Panel a, the two curves correspond to $h=0$ and $h=1, \omega=1$. In both cases the system is in its glassy phase. In panel $\mathrm{b}$ the two curves correspond to $\omega=1$ and $\omega=10$, both with an amplitude $h=1$, The lines are guides to the eye and they have the slope of the second part of the decay. $t_{w}=24$ for all curves displayed.

the time dependence of the energy density $e(t)=N^{-1} \sum_{i<j<k} J_{i j k} s_{i} s_{j} s_{k}$ and the magnetization density $m(t)=N^{-1} \sum_{i} s_{i}(t)$. The sizes $N=50$ and 150 have been used, together with a constant temperature $T=0.01$, in all simulations. The amplitude and the frequency of the magnetic field have been varied.

The interest of such an investigation is that the finite $N$ behavior of the model is accessible. The system is hence able to escape and visit the trapping states described in Section 2 in a finite times [18]. For that reason, the results presented in this section cannot be obtained in a mean-field approach.

In the absence of magnetic field, the energy density rapidly approaches its asymptotic value. At such a low temperature, the system reaches a metastable stable, characterized by a nearly zero magnetization density, and cannot escape within the numerical time window. Fig. 11 (left) illustrates this 'jammed' behavior. The value of the energy density depends on the initial conditions, which means that the sys- 

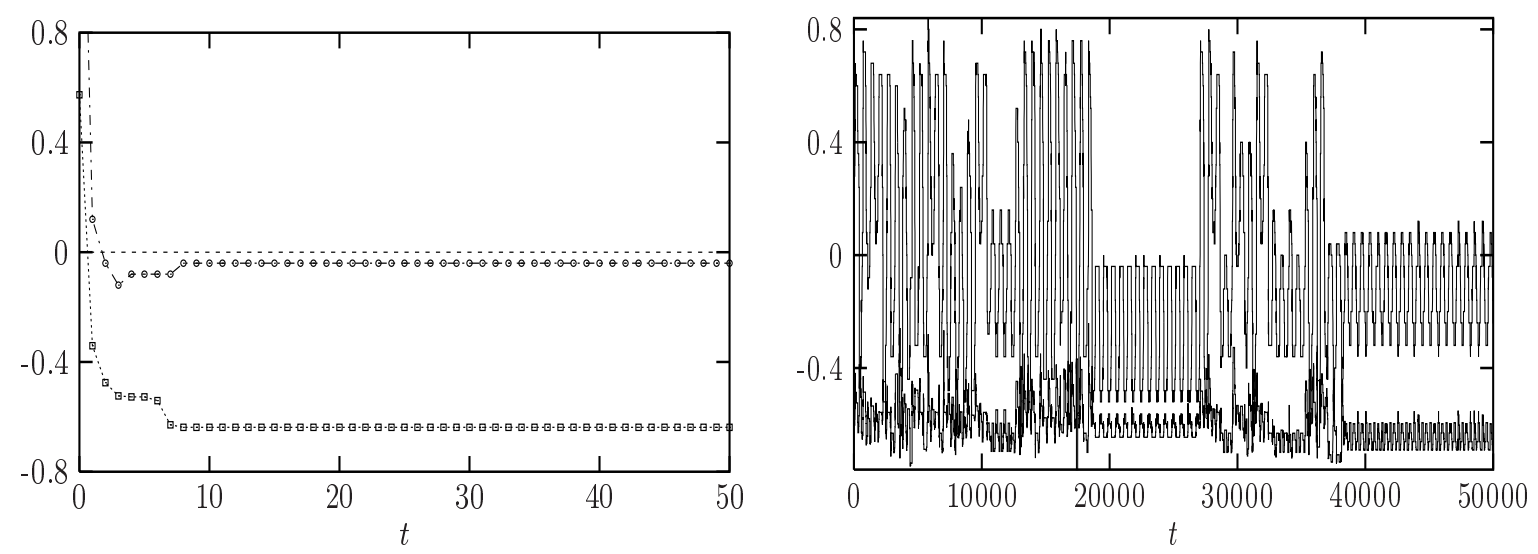

Figure 11: Evolution of the energy (bottom curve) and magnetization (top curve) densities without (left) and with (right) magnetic field. The parameters of the field are $h=2, \omega=0.01$. Note that the time range in the left figure is much shorter, since both $e(t)$ and $m(t)$ are constant for times $t>50$.

tem may be blocked in different trapping states when it starts from different initial conditions.

The influence of an ac magnetic field on this jammed behavior is evident in Fig. 11. Both the energy and the magnetization density are oscillating functions, and their behavior is intermittent. For some time windows, both quantities have an evolution which varies considerably from period to period. For other time windows, on the contrary, the different cycles are very similar. This can be interpreted as being due to the presence of the trapping ('jammed') states: the system usually evolves inside one of the trapping states, without escaping, and all the periods are equivalent. But from time to time, the driving force is able to make the system escape the state, and the evolution is very erratic until another trapping state is found.

This is confirmed in Fig. 12, where the energy density is represented as a function of $t$ for times of the form $t=n \tau$. The long horizontal plateaux are the moments where the system is jammed, whereas between the plateaux, the energy density changes values very rapidly. As in Ref. [18], we show in Fig. 12 that the time evolution of $e(t)$ is 'self-similar', in the sense that zooming on a time window makes smaller plateaux become visible while the overall evolution looks the same.

This intermittent behavior is clearly reminiscent of the behavior encountered in some granular experiments, where the powder is very slowly perturbed, like the ones performed by the Jussieu group [64]. In particular, it would be very interesting to perform a more careful analysis of the statistical properties of $e(t)$ like, e.g., measuring the statistics of trapping times [64]. We note also that this phenomenology is very similar to the one of the so-called 'trap model' [65], that has been extended by Head to describe the phenomenology of granular materials [36].

Finally, it is interesting to stress that even within one cycle of the field, the 

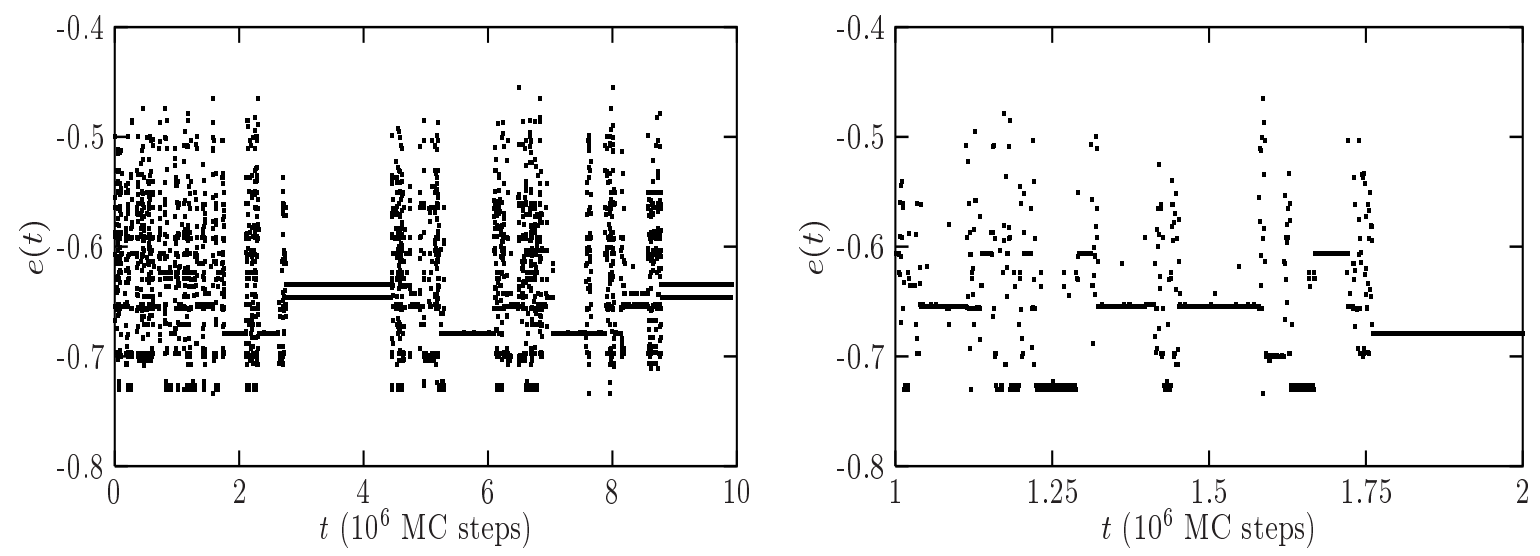

Figure 12: Same parameters as in Fig. 11. Evolution of the energy density with a magnetic field. Only one point per period is represented.

evolution is not regular at all. This can be nicely seen in Fig. 13, where the field $h(t)$ is represented as a function of the magnetization $m(t)$. This is the usual view of a hysteresis loop in ferromagnetic systems. It is clear that the shape of the loops is far from elliptic, and that the system evolves in steps, rather than continuously: this is analogous to the Barkhausen noise [66]. The overall shape of a cycle drastically depends on the fact that the system is trapped or not. It is beyond the scope of this paper to study these loops in detail, but we emphasize that this (mean-field) model could be an interesting starting point to study the Barkhausen noise, in the spirit of Ref. [66].

\section{Summary and link with granular matter}

Phase diagram. - Our findings concerning the behavior of glassy systems under a time-dependent driving force are summarized in Figs. 8 and 14. For any fixed frequency of the applied field, and a temperature $T<T_{c}$, where $T_{c}$ is the zero-drive glass transition temperature, there are two regimes: (I) At small drive, the system exhibits slow ("glassy") dynamics. (II) At large drive, there is no slow dynamics. There exists then a well-defined critical drive separating these two regimes. On the other hand, if we work at fixed drive and modify the angular velocity, the system undergoes a transition from a liquid-like phase at low (though non vanishing) frequency to a glass-like phase at high frequency. This result is displayed in Fig. 8.

The phase diagram in Fig. 14 is the analog to the $(T, \Gamma)$ plane of the threedimensional phase diagram proposed by Liu and Nagel [7]. We have then investigated its properties in detail, which had not been done so far in the case of a time-dependent driving force. Contrary to previous phenomenological modeling of the dynamics of granular matter assuming that the drive $\Gamma$ is related (in a possibly 

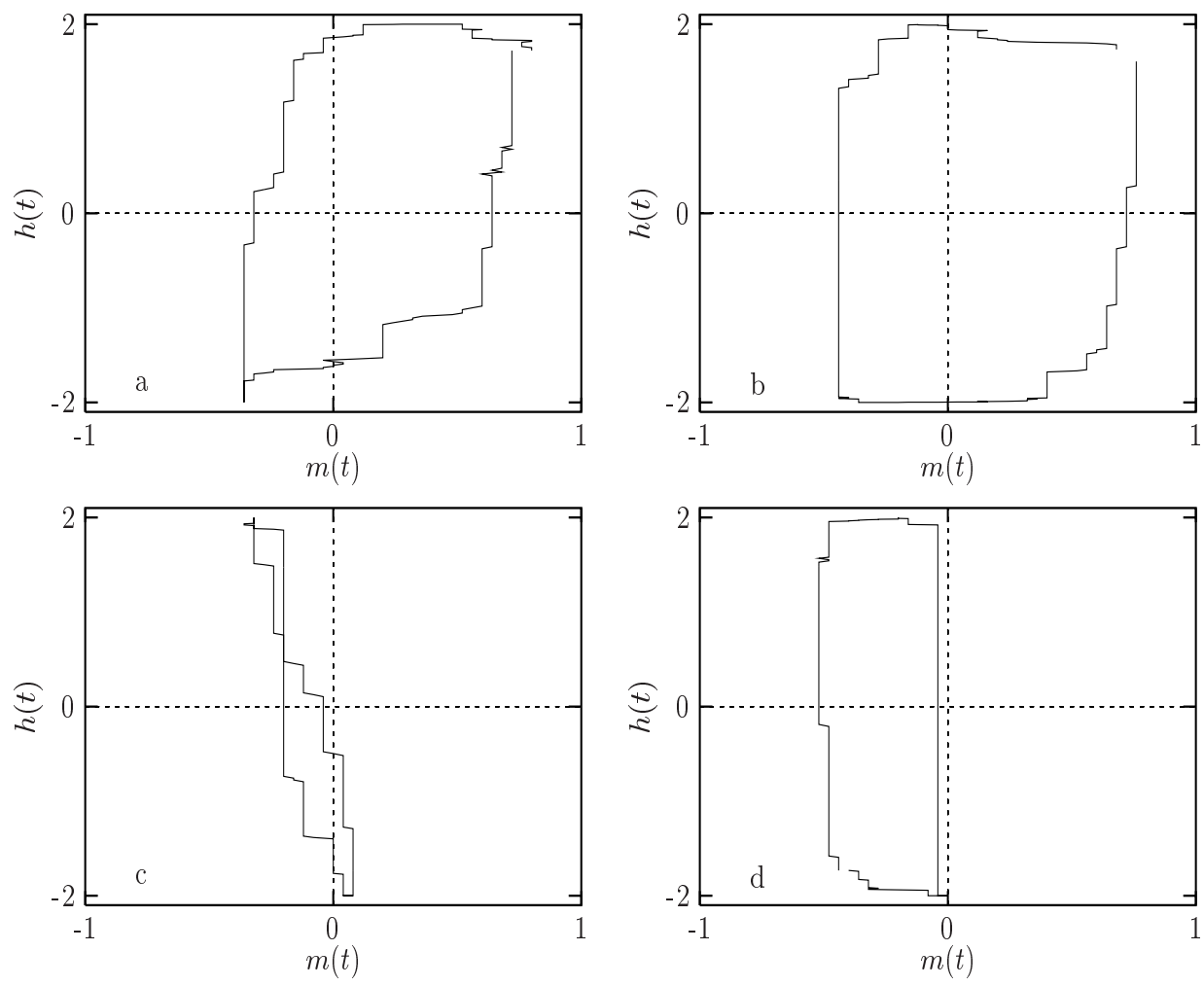

Figure 13: Hysteresis loops. Panels a and b correspond to the period of rapid motion while panels c and d correspond to the interval when the system is trapped. $N=50$, $T=0.01, h=2$ and $\omega=0.1$

nonlinear way) to the temperature $T$ of a glassy model borrowed from statistical mechanics, we propose here to study precisely the interplay between $T$ and $\Gamma$ as an intermediate step from glassy to granular materials. The conclusions we draw are then directly pertinent to gently driven granular materials or slow granular rheology.

We emphasize once again, that the model we have studied is not intended to describe granular matter in full microscopic detail, but rather to act as a source of inspiration for the interpretation of numerical and experimental results. It may also motivate new experimental measurements. Let us however briefly summarize its dynamic behavior in the context of existing data for granular matter.

One-time quantities. - In phase (I), one-time quantities (we have focused on the energy density) typically decay towards their asymptotic values as power laws.

It is experimentally established that the density $\rho(t)$ in a gently driven granular system exhibits a very slow relaxation [1]. The parallel can be drawn with the energy density $e(t)$ (or more precisely with $-1 / e(t)$ ) in glassy models. The seminal experiments at Chicago have exhibited a logarithmic relaxation of the density [2], as found in all subsequent works [4, 26, 27, 28, 29, 30, 31, 32, 34, 35, 36]. (Note that Ref. [2] explicitly excludes a power law behavior.) However, the recent experiment of Nicolas et al. [3] showed that the logarithmic law is not a universal behavior 


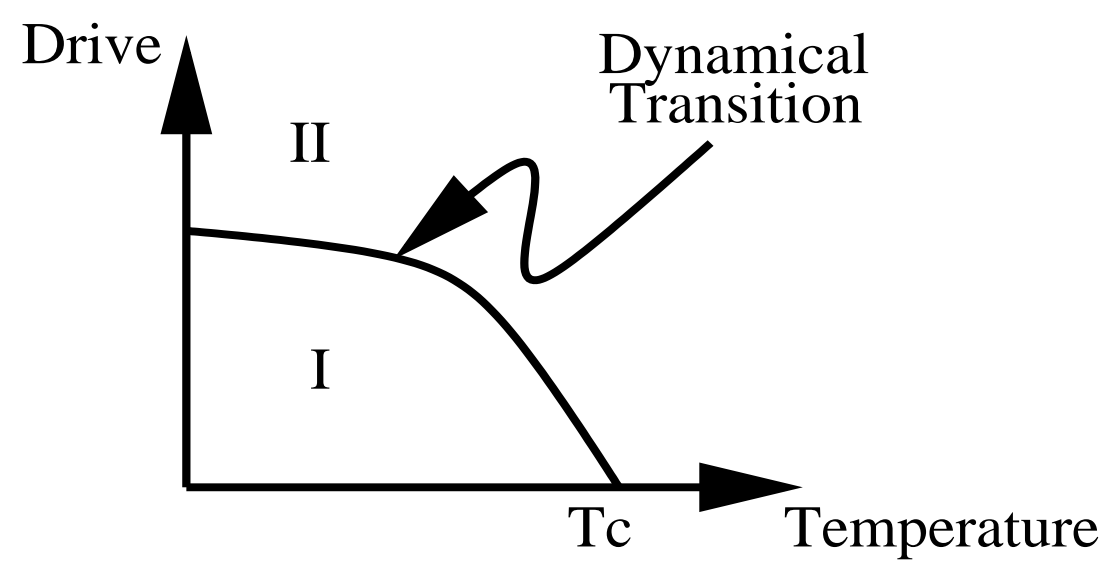

Figure 14: Temperature-Drive phase diagram of a glassy system. The vertical axis represents the typical amplitude of the driving force: its precise definition depends on the specific experiment considered; $T_{c}$ is the zero-drive glass transition temperature.

and that the time dependence may depend on the way the system is perturbed. From the theoretical point of view, one could prefer a power law decay for any one-time quantity since it does not involve an intrinsic time scale, whereas the logarithmic law $1 / \ln \left(t / t_{0}\right)$ explicitly introduces a time scale $t_{0}$. In this context, it is interesting to note that Barrat and Loreto [28], in their study of the Tetris model, have shown that the fitting parameter $t_{0}$ actually depends on the considered time window; qualitatively $t_{0} \propto t_{w}$. Head [36] had already emphasized the role of the time window on the fitting parameters, in particular on the asymptotic value of the density.

In the context of glassy systems, it is a well-known feature that the interpretation of data over a finite time-window is always delicate. A typical example is provided by the case of dipolar glasses where the dielectric constant for samples prepared with different cooling rates seems to approach different asymptotic values [15].

Two-time quantities. - In phase (I), the model we have studied also exhibits aging. For $p=2$, we find a $t / t_{w}$-scaling (in stroboscopic time) of the autocorrelation function while for $p \geq 3$ a much more careful numerical analysis is needed to determine this law. Other glassy models perturbed with ac-forces may lead to other aging scalings. Generally speaking, theoretical arguments [11] indicate that one can expect the following behavior for any two-time function $F\left(t, t_{w}\right)$

$$
F\left(t, t_{w}\right) \sim \mathcal{F}\left(\frac{h(t)}{h\left(t_{w}\right)}\right)
$$

in a given time-scale. $\mathcal{F}$ and $h$ are two model/system-dependent scaling functions. More complicated scalings, such as a sum of different terms like Eq. (28) implying several different time scales in the problem, are also possible.

Two-time quantities such as the density-density correlation function have been numerically studied, clearly demonstrating that TTI is lost [27, 31, 28]: the Tetris 
model exhibits a $\ln (t) / \ln \left(t_{w}\right)$ behavior [27, 28], whereas the parking lot model shows a $t / t_{w}$ aging behavior [31].

An experimental determination of the scaling of two-time quantities will be useful to discriminate among the different models proposed to describe granular matter. This has already been proposed in Refs. [27, 28, 31] and amounts to an experimental determination of the function $h(t)$ involved in Eq. (28). Such experimental determinations in glassy systems are by now numerous. They have emphasized on the one hand the universality of the aging phenomena in many microscopically different systems [12, 13, 14, 15, 16], but on the other hand they have also revealed subtle differences between them [11, 13].

Fluctuation-dissipation theorem violations. - Our study has shown that modifications of the FDT, rather similar to those observed for aging glassy systems, also arise in glassy models driven by time-dependent forces. The parallel with granular materials suggests that this may also happen in driven powders.

The parametric plot we have built clearly shows that driven glassy systems have two distinct time scales, which we interpret as in Refs. [8, 9]. A fast one, which is essentially independent of the waiting time, represents the individual motion of grains: no effective temperature, in the sense of Eq. (9), can be here defined. Since the model we have used is itself thermal (it uses a Langevin equation with a thermal noise) the fast motion has a reminiscence of the temperature of the bath (that may be zero). In a more realistic model of granular matter, it is likely that the fast motions will be completely athermal. On the contrary, the long time scale growing with the waiting time and giving rise to the $t / t_{w}$-relaxation, represents large structural rearrangements in the powder. Our results indicate that these slow degrees of freedom are thermalized at a well-defined effective temperature. We are confident that this result also holds in realistic models of granular matter.

Again, numerical and experimental work could be used to check these predictions in granular materials. We have shown that the two models studied here have different FDT violations: the $p=2$ case has an infinite effective temperature, whereas the $p \geq 3$ models have a single finite effective temperature. Other glassy models (for instance mean-field spin glasses) have been shown to have a hierarchy of effective temperatures when they are undriven, and shall probably keep this behavior under driving forces 67. We conjecture that granular materials fall in the $p \geq 3$ category, by analogy with structural glasses. This prediction has to be experimentally and numerically tested. It is not unlikely that different microscopic models lead to different FDT-violations; such a numerical determination could once again discriminate between them.

To study FDT violations during the compaction experiment, one has to compute, separately, the correlation function between two observables and its conjugated response function. In experiments, for instance, the Nyquist relation is checked through dielectric measurements [14, 68]. In granular materials, a natural choice is to check the Einstein relation between self-diffusion and mobility of the grains. The results obtained in Refs. 69, 70 are then very encouraging, although the driving field is not time-dependent in these studies. Nicodemi studied instead the fluctuation-dissipation relation between height-height displacement and its conju- 
gated integrated response to small shaking amplitude perturbations in the Tetris model [71]. In the low density regime (fluide-like), after a short transient, these two quantities are related by the usual FDT. In the high density regime, the relation between these quantities shows much stronger deviations from FDT than the model we studied here. This difference may be due to the kind of perturbation used by Nicodemi. For a thermal system, the procedure in Ref. [71] is indeed similar to measuring the energy response to a temperature change: The resulting FDT properties are found to be of an unusual type [72 also in this case. However, the notion of an effective temperature [52] implies that the FDT relations of all observables evolving in the same time-scale should be identical. As suggested in Refs. [28, 69], an interesting open question is: does a measurement in the Tetris model done with a more standard infinitesimal perturbation lead to the same FDT relation? This point deserves a more detailed investigation in this and other glassy models.

The dynamic transition, and the regime (II). - We have found a well-defined transition in the phase diagram of Fig. 14. This is in complete agreement with experimental and numerical observations of the existence of a critical value of the drive $\Gamma^{\star}$, below which glassy effects may be observed. The existence of this dynamical transition provides a very natural context for the interpretation of the numerous non-equilibrium effects encountered in granular matter. Indeed, the transition line in the Fig. 14 links the standard glass transition arising at $T=T_{c}, \Gamma=0$ in the glassy model to the dynamical transition arising at $T=0, \Gamma=\Gamma^{\star}$ in the driven dynamics of athermal systems. In our opinion, this result gives a nice theoretical

support to the glasses/granular analogy, making a deep connection between the two situations.

We wish to emphasize that it was not evident a priori that the transition could survive a finite time-dependent driving force. This is one of the main results of this paper and it has to be confronted to the very different effect of shear-like forces. Indeed, an infinitesimal shear-like perturbation, is enough to introduce a finite timescale in this model, and aging is hence interrupted [18, 21.

The liquid regime (II) is less interesting in the present context, since no slow dynamics is present. Let us stress that the system is still strongly driven, and hence no 'equilibrium' state, in the sense of statistical mechanics, is reached. In particular, no effective FDT temperature can be defined: the system is completely athermal. The very existence of an effective temperature relies indeed on the existence of two well-separated time scales allowing the slow degrees of freedom to thermalize and the susceptibility / correlation parametric curves of Fig. 9-a are not very useful to describe this non-equilibrium situation.

\section{Conclusion: towards a thermodynamical descrip- tion of the slow granular rheology?}

The interpretation of FDT violations in terms of an effective temperature $T_{\mathrm{EFF}}$ has been developed in Ref. [52]. Having a well-defined concept of temperature is a crucial first step for a thermodynamical description of granular materials [73]. It is 
then important to discuss the possibility of a link with previous thermodynamical concepts in the granular literature.

The notion of a 'granular temperature' has been introduced to extend thermodynamics to strongly perturbed powders [1]. In analogy with the kinetic gas theory, it has been assumed that distribution of the grain velocity $v$ is Maxwellian and via the equipartition relation $T \propto\left\langle v^{2}\right\rangle$. We have been concerned with the weakly perturbed regime, where the dynamics is slow. This hydrodynamic definition is not supposed to be relevant in this case. This is also well known in the field of glasses where nontrivial effective temperatures are known to exist while the kinetic energy is an observable that very rapidly equilibrates with its environment, leading to a kinetic temperature that coincides with the temperature of the bath. Very recently [74], an attempt to extend the hydrodynamic theory to a regime where the grains are not fluidized has been proposed, also using the concept of kinetic granular temperature. It would then be very interesting to try to understand the two concepts in a unified way.

More related to ours is the approach of Mehta et al. [8], discussed in the introduction. This phenomenological two-step, two-temperature model finds a nice justification within the scenario emerging from our results. This model was in fact inspired by the illuminating work initiated in the late 80's by Edwards and coworkers [38. They have postulated thermodynamic relations in analogy with the usual 'thermal' thermodynamics, where the volume $V$ plays the role of the internal energy $U$. In this approach, the entropy is the logarithm of the number of configurations with volume $V$. The so-called compactivity, $X$, is the analog of the temperature and it then defined as [38]

$$
\frac{1}{X} \equiv \frac{\partial S}{\partial V}
$$

still by analogy with the usual definition $T^{-1} \equiv \partial S / \partial U$. The connection between the compactivity $X$ and the FDT temperature $T_{\mathrm{EFF}}$ discussed in this paper has already been explored [10, 57, 58, 62, 71]. Crucially for our study, Edwards' definition of the compactivity coincides with the (asymptotic) FDT effective temperature defined in Eq. (14). This result holds for mean-field models like the one discussed here [62], as well as in the lattice gas model studied in Ref. [57]. However, these studies have been done without an external forcing. It would be very interesting to extend the numerical analysis of Ref. [57] to the same model now driven by an ac force.

We have obtained here that the existence of an effective temperature for the slow decay resists a finite ac force. This supports the conjecture that the definitions (14) and (29) may be of fundamental interest for the study of glassy/granular materials. Computing them in realistic models is then a challenge for future research [57, 58, 62, 75]. In this respect, very recent works [76] studying spin models on random graphs may give some insights on the role played by metastable states, which can have an important influence on the dynamics, as we showed in Section 4.3

In conclusion, we have found that the study of driven glassy systems provides a theoretical framework to understand the slow granular rheology. The existence of 
a dynamical transition justifies well the use of 'modified glassy models' to describe granular materials. The existence of an effective temperature for the slow degrees of freedom provides in particular a nice theoretical basis to previous 'thermal' models for granular matter, and to the seminal approach of Edwards.

\section{Acknowledgments}

We wish to thank J.-L. Barrat, E. Kolb, J. Kurchan, F. Restagno, M. Sellitto, L. Vanel and J. Wittmer for very useful discussions.

\section{References}

[1] H. M. Jaeger, S. R. Nagel, and R. P. Behringer, Rev. Mod. Phys. 68, 1259 (1996); H. M. Jaeger and S. R. Nagel, Science 255, 1523 (1992); P. G. de Gennes, Rev. Mod. Phys. 71, S374 (1999).

[2] J. B. Knight, C. G. Fandrich, C. N. Lau, H. M. Jaeger and S. R. Nagel, Phys. Rev. E 51, 3957 (1995); E. R. Nowak, J. B. Knight, E. Ben-Naim, H. M. Jaeger and S. R. Nagel, Phys. Rev. E 57, 1971 (1998).

[3] M. Nicolas, P. Duru and O. Pouliquen, Eur. Phys. J. E 3, 309 (2000).

[4] H. M. Jaeger, C.-H. Liu and S. R. Nagel, Phys. Rev. Lett. 62, 40 (1989).

[5] É. Falcon, R. Wunenburger, P. Évesque, S. Fauve, C. Chabot, Y. Garrabos and Daniel Beysens, Phys. Rev. Lett. 83, 440 (1999), and references therein.

[6] S. F. Edwards and D. V. Grinev, Proceedings of "Jamming and Rheology: constrained dynamics on microscopic and macroscopic scales", ITP, Santa Barbara, 1997, preprint cond-mat/9905114.

[7] A. J. Liu and S. R. Nagel, Nature 396, 21 (1998).

[8] A. Mehta, R. J. Needs and S. Dattagupta, J. Stat. Phys. 68, 1131 (1992).

[9] A. Mehta and G. C. Barker, J. Phys.: Condens. Matter 12, 6619 (2000); G. C. Barker and A. Mehta, Phys. Rev. E 47, 184 (1993); A. Mehta and G. C. Barker, Phys. Rev. Lett. 67, 394 (1991); G. C. Barker and A. Mehta, Phys. Rev. A 45, 3435 (1992).

[10] J. Kurchan, Rheology, and how to stop aging, Proceedings of "Jamming and Rheology: constrained dynamics on microscopic and macroscopic scales", ITP, Santa Barbara, 1997, preprint cond-mat/9812347 and J. Phys.: Condens. Matter 12, 6611 (2000).

[11] J.-P. Bouchaud, L.F. Cugliandolo, J. Kurchan and M. Mézard in Spin Glasses and Random Fields, Ed.: A. P. Young (World Scientific, Singapore, 1998). 
[12] L. C. E. Struik, Physical aging in amorphous polymers and other materials (Elsevier, Houston, 1978); L. Bellon, S. Ciliberto and C. Laroche, Europhys. Lett. 51, 551 (2000).

[13] E. Vincent, J. Hammann, M. Ocio, J.-Ph. Bouchaud and L. F. Cugliandolo, in Complex behavior of glassy systems, Ed.: M. Rubi (Springer Verlag, Berlin, 1997); P. Nordblad and P. Svendlidh, in Spin Glasses and Random Fields, Ed.: A. P. Young (World Scientific, Singapore, 1998).

[14] R. L. Leheny and S. R. Nagel, Phys. Rev. B 57, 5154 (1998); T. Grigera and N. Israeloff, Phys. Rev. Lett. 83, 5038 (1999).

[15] F. Alberici-Kious, J.-Ph. Bouchaud, L. F. Cugliandolo, P. Doussineau and A. Levelut, Phys. Rev. Lett. 81, 4987 (1998).

[16] M. Kroon, G. H. Wegdam and R. Sprick, Phys. Rev. E 54, 6541 (1996); D. Bonn et al., Europhys. Lett. 45, 52 (1998); A. Knaebel et al., Europhys. Lett. 52, 73 (2000); L. Cipelletti et al., Phys. Rev. Lett. 84, 2275 (2000).

[17] A. J. Bray, Adv. Phys. 43, 357 (1994).

[18] L. F. Cugliandolo, J. Kurchan, P. Le Doussal and L. Peliti, Phys. Rev. Lett. 78, 350 (1997).

[19] A. Onuki, J. Phys. C 9, 6119 (1997).

[20] R. G. Larson, The Structure and Rheology of Complex Fluids (Oxford University Press, New York, 1999).

[21] L. Berthier, J.-L. Barrat and J. Kurchan, Phys. Rev. E 61, 5464 (2000).

[22] P. Sollich, Phys. Rev. E 58, 738 (1998); S. M. Fielding, P. Sollich and M. E. Cates, J. of Rheology 44, 323 (2000).

[23] C. Dérec, A. Ajdari, F. Lequeux, Faraday Discuss. 112, 195 (1999); C. Dérec, A. Ajdari and F. Lequeux, preprint (2000).

[24] C. Josserand, A. Tkachenko, D. M. Mueth and H. M. Jaeger, Phys. Rev. Lett. 85, $3632(2000)$.

[25] G. D'Anna, preprint cond-mat/0006323.

[26] A. Coniglio and H.J. Herrmann, Physica A 225, 1 (1996); M. Nicodemi, A. Coniglio, H.J. Herrmann, Phys. Rev. E 55, 3962 (1997); J. Phys. A 30, L379 (1997).

[27] M. Nicodemi and A. Coniglio, Phys. Rev. Lett. 82, 916 (1999); A. Coniglio and M. Nicodemi, Phys. Rev. E 54, 2812 (1999); A. Coniglio and M. Nicodemi, J. Phys. C 12, 6601 (2000). 
[28] A. Barrat and V. Loreto, J. Phys. A 33, 4401 (2000); A. Barrat and V. Loreto, preprint cond-mat/0006413.

[29] M. Sellitto and J. J. Arenzon, Phys. Rev. E 62, 7793 (2000).

[30] T. Boutreux and P. G. de Gennes, Physica A 244, 59 (1997).

[31] A. J. Kolan, E. R. Nowak and A. V. Tkachenko, Phys. Rev. E 59, 3094 (1999); J. Talbot, G. Tarjus and P. Viot, Phys. Rev. E 61, 5429 (2000); preprint condmat/0008183.

[32] S. J. Linz, Phys. Rev E 54, 2925 (1996).

[33] D. C. Hong, S. Yue, J. K. Rudra, M. Y. Choi and Y. W. Kim, Phys. Rev. E 50, 4123 (1994).

[34] K. L. Gavrilov, Phys. Rev. E 58, 2107 (1998).

[35] D. A. Head and G. J. Rodgers, J. Phys. A 31, 107 (1998).

[36] D. A. Head, Phys. Rev E 62, 2439 (2000).

[37] S. F. Edwards and D. V. Grinev, Phys. Rev. E 58, 4758 (1998).

[38] S. F. Edwards, in Disorder in condensed matter physics, (Oxford Science Pubs., 1991); S. F. Edwards, in Granular Matter: An interdisciplinary Approach, Ed.: A. Mehta (Springer Verlag, New York, 1994), and Refs. therein.

[39] J.-L. Barrat and L. Berthier, Phys. Rev. E 63, 012503 (2001).

[40] Ac-perturbations of short duration, typically a few cycles, have been experimentally used to probe the dynamics of glassy materials in, e.g., non resonant hole-burning experiments [41, 42]. A careful study of the dynamics of glassy systems under a permanent ac-perturbation is, as far as we know, still lacking.

[41] M. T. Cicerone and M. D. Ediger, J. Chem. Phys. 103, 5684 (1995); R. Böhmer et al, J. Non-Cryst. Solids 235-237 I-9 (1998) and Refs. therein.

[42] L. F. Cugliandolo and J. L. Iguain, Phys. Rev. Lett. 85, 3448 (2000).

[43] T. R. Kirkpatrick and D. Thirumalai, Phys. Rev. B 36, 5388 (1987).

[44] A. Crisanti and H.-J. Sommers, Z. Phys. B 87, 341 (1992).

[45] J. M. Kosterlitz, D. J. Thouless and R. C. Jones, Phys. Rev. Lett. 36, 1217 (1976).

[46] P. Shukla and S. Singh, Phys. Rev. B 23, 4661 (1981). S. Ciuchi and F. de Pasquale, Nucl. Phys. B 300, 31 (1988).

[47] L. F. Cugliandolo and D. S. Dean, J. Phys. A 28, 4213 (1995); W. Zippold, R. Kuehn and H. Horner, Eur. Phys. J. B 13, 531 (2000). 
[48] L. F. Cugliandolo and D. S. Dean, J. Phys. A 28, L453 (1995).

[49] M. Mézard and G. Parisi, J. Chem. Phys. 111, 1076 (1999).

[50] L. F. Cugliandolo and J. Kurchan, Phys. Rev. Lett. 71, 173 (1993).

[51] B. Derrida, Phys. Rev. B 24, 2613 (1981); D. J. Gross and M. Mézard, Nucl. Phys. B 240, 431 (1984).

[52] L. F. Cugliandolo, J. Kurchan and L. Peliti, Phys. Rev. E 55, 3898 (1997).

[53] L. F. Cugliandolo and J. Kurchan, J. Phys. A 27, 5749 (1994).

[54] S. A. Langer and A. J. Liu, Europhys. Lett. 49, 68 (2000).

[55] P. H. Stillinger and T. A. Weber, Science 255, 983 (1984).

[56] W. Kob, F. Sciortino and P. Tartaglia, Europhys. Lett. 49, 590 (2000); G. Biroli and R. Monasson, Europhys. Lett. 50, 155 (2000).

[57] A. Barrat, J. Kurchan, V. Loreto and M. Sellitto, Phys. Rev. Lett. 85, 5034 (2000); preprint cond-mat/0011492.

[58] A. Coniglio and M. Nicodemi, cond-mat/0010191.

[59] L. F. Cugliandolo, D. S. Dean and J. Kurchan, Phys. Rev. Lett. 79, 2168 (1997).

[60] W. Götze, in Liquids, Freezing and Glass Transition, Eds.: J.P. Hansen, D. Levesque and J. Zinn-Justin, Les Houches 1989, (North Holland, Amsterdam, 1991); W. Götze and L. Sjögren, Rep. Prog. Phys. 55, 241 (1992); W. Götze, J. Phys. Condens. Matter 11, A1 (1999).

[61] J. Kurchan, G. Parisi and M. A. Virasoro, J. Phys. I (France) 3, 1819 (1993); A. Barrat, R. Burioni and M. Mézard, J. Phys. A 29, L81 (1996); A. Cavagna, I. Giardina and G. Parisi, Phys. Rev. B 57, 11251 (1998).

[62] R. Monasson, Phys. Rev. Lett. 75, 2847 (1995); R. Monasson and O. Pouliquen, Physica A 236, 395 (1997).

[63] A. Cavagna, J. P. Garrahan and I. Giardina, J. Phys. A 32, 711 (1999).

[64] E. Kolb, E. Clément, Eur. J. Phys. B (1999); L. Vanel and É. Clément, Eur. Phys. J. B 11, 525 (1999); L. Vanel, Thèse de Doctorat de l'Université Paris 6 (1999).

[65] For a review, see J.-P. Bouchaud, Soft and fragile matter, nonequilibrium, metastability and flow, Eds.: M. E. Cates and M. R. Evans (Institue of Physics Publishing, Bristol, 2000). 
[66] J. P. Sethna, O. Perkovic and K. A. Dahmen, in Scale Invariance and Beyond, Eds.: B. Dubrulle, F. Graner, and D. Sornette, (Springer, Berlin, 1997), and Refs. therein.

[67] L. Berthier, J.-L. Barrat and J. Kurchan, Phys. Rev. E 63, 016105 (2001).

[68] L. Bellon, S. Ciliberto and C. Laroche, Europhys. Lett. (in press), preprint cond-mat/0008160.

[69] M. Sellitto, preprint cond-mat/0012273.

[70] H. Makse (unpublished).

[71] M. Nicodemi, Phys. Rev. Lett. 82, 3734 (1999).

[72] S. M. Fielding and P. Sollich (unpublished).

[73] See also, in the context of glassy systems: T. M. Nieuwenhuizen, Phys. Rev. E 61, 267 (2000) and Refs. therein; S. Franz and M. A. Virasoro, J. Phys. A 33, 891 (2000).

[74] W. Losert, L. Bocquet, T. C. Lubensky and J. P. Gollub, Phys. Rev. Lett. 85, 1428 (2000); L. Bocquet, W. Losert, T. C. Lubensky and J. P. Gollub, preprint cond-mat/0012356.

[75] G. Biroli and J. Kurchan, preprint cond-mat/0005499.

[76] J. Berg and A. Mehta, preprint cond-mat/0012416; D. S. Dean and A. Lefevre, preprint cond-mat/0101166. 